\title{
THE LUMINOSITY FUNCTION OF FERMI-DETECTED FLAT-SPECTRUM RADIO QUASARS
}

\author{
M. Ajello ${ }^{1}$, M. S. Shaw ${ }^{1}$, R. W. Romani ${ }^{1}$, C. D. Dermer ${ }^{2}$, L. Costamante $^{1}$, O. G. King ${ }^{3}$, W. Max-Moerbeck ${ }^{3}$,

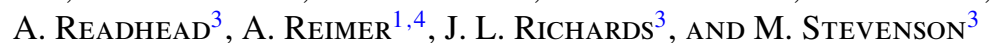 \\ ${ }^{1}$ W. W. Hansen Experimental Physics Laboratory, Kavli Institute for Particle Astrophysics and Cosmology, Department of Physics and SLAC National Accelerator \\ Laboratory, Stanford University, Stanford, CA 94305, USA; majello@slac.stanford.edu, msshaw@ stanford.edu, rwr@ astro.stanford.edu \\ ${ }^{2}$ Space Science Division, Naval Research Laboratory, Washington, DC 20375-5352, USA \\ ${ }^{3}$ Cahill Center for Astronomy and Astrophysics, California Institute of Technology, Pasadena, CA 91125, USA \\ ${ }^{4}$ Institut für Astro- und Teilchenphysik and Institut für Theoretische Physik, Leopold-Franzens-Universität Innsbruck, A-6020 Innsbruck, Austria \\ Received 2011 October 15; accepted 2012 March 28; published 2012 May 11
}

\begin{abstract}
Fermi has provided the largest sample of $\gamma$-ray-selected blazars to date. In this work we use a complete sample of flat spectrum radio quasars (FSRQs) detected during the first year of operation to determine the luminosity function (LF) and its evolution with cosmic time. The number density of FSRQs grows dramatically up to redshift $\sim 0.5-2.0$ and declines thereafter. The redshift of the peak in the density is luminosity dependent, with more luminous sources peaking at earlier times; thus the LF of $\gamma$-ray FSRQs follows a luminosity-dependent density evolution similar to that of radio-quiet active galactic nuclei. Also, using data from the Swift Burst Alert Telescope we derive the average spectral energy distribution (SED) of FSRQs in the $10 \mathrm{keV}-300 \mathrm{GeV}$ band and show that there is no correlation between the luminosity at the peak of the $\gamma$-ray emission component and its peak frequency. Using this luminosity-independent SED with the derived LF allows us to predict that the contribution of FSRQs to the Fermi isotropic $\gamma$-ray background is $9.3_{-1.0}^{+1.6} \%$ ( $\pm 3 \%$ systematic uncertainty) in the $0.1-100 \mathrm{GeV}$ band. Finally we determine the LF of unbeamed FSRQs, finding that FSRQs have an average Lorentz factor of $\gamma=11.7_{-2.2}^{+3.3}$, that most are seen within $5^{\circ}$ of the jet axis, and that they represent only $\sim 0.1 \%$ of the parent population.
\end{abstract}

Key words: cosmology: observations - diffuse radiation - galaxies: active - galaxies: jets - gamma rays: diffuse background - surveys

Online-only material: color figures

\section{INTRODUCTION}

The detection of luminous quasars at redshift $>6$ (e.g., Fan et al. 2003; Willott et al. 2010) provides evidence of supermassive black hole (SMBH) formation in the first $1 \mathrm{Gyr}$ of cosmic time. There are appreciable challenges to forming (see, e.g., Wyithe \& Loeb 2003; Volonteri \& Rees 2005; Begelman et al. 2006; Mayer et al. 2010) and fueling (see Kauffmann \& Haehnelt 2000; Wyithe \& Loeb 2003; Croton et al. 2006) these objects at such early times, although it is widely believed that strong accretion can be initiated by major mergers (see Kauffmann \& Haehnelt 2000; Wyithe \& Loeb 2003; Croton et al. 2006).

Blazars represent an extreme manifestation of such active galactic nucleus (AGN) activity, with radiation along Earth's line of sight dominated by a relativistic jet. It is, as yet, unclear how such jet activity connects with the more isotropically emitted bulk accretion luminosity. For example according to Blandford \& Znajek (1977), the energy stored in a black hole's spin can be extracted in the form of a relativistic jet. Thus, blazar evolution may be connected with the cosmic evolution of the spin states of massive black holes. Radio-loud (RL, jet dominated) blazars have been seen at redshifts as high as $z=5.5$ (Romani 2006), and it is plausible that major mergers, more frequently experienced in the early universe, might preferentially produce maximally rotating black holes (e.g., see Escala et al. 2004; Dotti et al. 2007).

Thus the study of RL AGN, blazars, with strong relativistically beamed jets can provide a method to study jet activity, $\mathrm{BH}$ spin, and major merger events. This can be done by determining the luminosity function (LF) of blazars (essentially the number of blazars per comoving volume element within a certain luminosity range) and its evolution with redshift. The Fermi Gamma-ray Space Telescope provides one of the largest data sets with which to study the properties of blazars. Thanks to its sensitivity and uniform coverage of the sky, Fermi has detected hundreds of blazars from low redshifts out to $z=3.1$ (Ackermann et al. 2011).

The LF of blazars also allows us to evaluate their contribution to the diffuse backgrounds and to determine their relationship with the parent population (Ajello et al. 2008b; Inoue 2011). Blazars have been extensively studied at radio (Dunlop \& Peacock 1990; Wall et al. 2005), soft X-ray (Giommi \& Padovani 1994; Rector et al. 2000; Wolter \& Celotti 2001; Caccianiga et al. 2002; Beckmann et al. 2003; Padovani et al. 2007), and $\mathrm{GeV}$ energies (Hartman et al. 1999). It seems clear that flat spectrum radio quasars (FSRQs) evolve positively (i.e., there were more blazars in the past, Dunlop \& Peacock 1990) up to a redshift cutoff which depends on luminosity (e.g., Padovani et al. 2007; Wall 2008; Ajello et al. 2009a). In this respect FSRQs evolve similarly to the population of X-ray-selected, radio-quiet AGNs (Ueda et al. 2003; Hasinger et al. 2005; La Franca et al. 2005). On the other hand, the evolution of the other major class of Fermi-detected AGN, BL Lac objects, and their relation to FSRQs, remains a matter of debate, with claims of no evolution (Caccianiga et al. 2002; Padovani et al. 2007) or even negative evolution (e.g., Rector et al. 2000; Beckmann et al. 2003). Samples with larger redshift completeness fractions are needed to validate these claims.

In this work, we report on the LF of FSRQs detected by Fermi in its first year of operation. There have been attempts in the past (e.g., Chiang et al. 1995) to characterize the evolution of $\gamma$-ray AGNs, starting from the EGRET sample (Hartman et al. 1999). One challenge was the small sample size and redshift 
incompleteness of the EGRET set. Often it was assumed that the $\gamma$-ray-detected blazars had an LF following that of another band, e.g., radio- or X-ray-selected blazars. The results reported in, e.g., Stecker \& Salamon (1996), Narumoto \& Totani (2006), Inoue \& Totani (2009), and Stecker \& Venters (2011) follow this approach. Alternatively, an LF may be estimated from the $\gamma$-ray sample directly (Chiang \& Mukherjee 1998; Mücke \& Pohl 2000; Dermer 2007; Bhattacharya et al. 2009), although only a small $(\sim 60)$ blazar sample including both BL Lac objects and FSRQs was available (from EGRET data) with acceptable incompleteness.

We report here on a detailed LF measured from a sample of $186 \gamma$-ray-selected FSRQs detected by Fermi. The work is organized as follows. Sections 2 and 3 describe the properties of the sample used and the method employed to determine the LF of blazars. The LF of FSRQs is derived in Section 4. In Section 5, the spectral energy distributions (SEDs) of Fermi's FSRQs are analyzed in detail, testing for possible correlations of the peak energy with the peak luminosity. The contribution of FSRQs to the isotropic gamma-ray background ${ }^{5}$ (IGRB; see Abdo et al. 2010e) is determined and discussed in Section 6. Throughout this paper, we assume a standard concordance cosmology $\left(H_{0}=\right.$ $71 \mathrm{~km} \mathrm{~s}^{-1} \mathrm{Mpc}^{-1}$ and $\left.\Omega_{M}=1-\Omega_{\Lambda}=0.27\right)$.

\section{THE SAMPLE}

The First Fermi Large Area Telescope (LAT) Catalog (1FGL; Abdo et al. 2010a) reports on more than 1400 sources detected by Fermi-LAT during its first year of operation. The first LAT AGN catalog (1LAC; Abdo et al. 2010g) associates 700 of the high-latitude 1 FGL sources $\left(|b| \geqslant 10^{\circ}\right)$ with AGNs of various types, most of which are blazars. The association method is probabilistic, by comparing the chance probability for gammaray sources to be found in the vicinity of sources in some underlying radio, optical, and X-ray AGN catalogs with the probability of chance associations. Among these catalogs, the most important ones are the Combined Radio All-sky Targeted Eight GHz Survey (CRATES; Healey et al. 2007), the Candidate Gamma-Ray Blazar Survey (CGRaBS; Healey et al. 2008), and the Roma-BZCAT (Massaro et al. 2009), which is a catalog of blazars identified at all frequencies. Here, we adopt the 1LAC associations with probability $>0.8$, i.e., $<20 \%$ chance of being a false positive, although most of the identifications are at the 0.95 or better level (Abdo et al. 2010g). The subset of these 1LAC AGNs used in the present analysis are those sources at $|b|>15^{\circ}$ detected by the pipeline developed by Abdo et al. (2010f) with a test statistic (TS) significance greater than 50 (corresponding to a significance of $\gtrsim 7 \sigma$; see, e.g., Mattox et al. 1996). For this sample, we have produced a set of Monte Carlo simulations that can be used to determine and account for the selection effects. This sample contains 483 objects, 186 of which are classified as FSRQs. The faintest identified FSRQ has a $100 \mathrm{MeV}-100 \mathrm{GeV}$ band flux of $F_{100} \approx 10^{-8}$ photons $\mathrm{cm}^{-2} \mathrm{~s}^{-1}$. To limit the incompleteness (i.e., the fraction of sources without an association) we apply this as a flux limit to the sample of 483 objects, resulting in a full sample of 433 sources of which 29 (i.e., 7\%) do not have associated counterparts. Note that all of the associations with an FSRQ designation have spectroscopically measured redshifts either from the literature or from recent spectroscopic studies (Shaw et al. 2012).

\footnotetext{
5 The isotropic gamma-ray background refers to the isotropic component of the Fermi sky (Abdo et al. 2010e) and as such might include components generated locally (e.g., Keshet et al. 2004) and components of truly extragalactic origin.
}

Table 1

Composition of the $|b| \geqslant 15^{\circ}$, TS $\geqslant 50, F_{100} \geqslant 10^{-8}$ photons $\mathrm{cm}^{-2} \mathrm{~s}^{-1}$ Sample Used in This Analysis

\begin{tabular}{lc}
\hline \hline CLASS & No. of objects \\
\hline Total & 433 \\
FSRQs & 186 \\
BL Lac objects & 157 \\
Pulsars & 28 \\
Other $^{\mathrm{a}}$ & 16 \\
Radio associations $^{\mathrm{b}}$ & 17 \\
Unassociated sources & 29 \\
\hline
\end{tabular}

Notes.

${ }^{a}$ Includes starburst galaxies, LINERS, narrow-line Seyfert 1 objects, and Seyfert galaxy candidates.

b Fermi sources with a radio counterpart, but no optical type or redshift measurement.

The composition of this sample is reported in Table 1 . The 186 FSRQs detected by Fermi with TS $\geqslant 50,|b| \geqslant 15^{\circ}$, and $F_{100} \geqslant 10^{-8}$ photons $\mathrm{cm}^{-2} \mathrm{~s}^{-1}$ constitute the sample that will be used in this analysis.

\section{METHOD}

A classical approach to derive the LF is based on the $1 / V_{\text {MAX }}$ method of Schmidt (1968) applied to redshift bins. However, this method is known to introduce bias if there is significant evolution within the bins. Moreover, given our relatively small sample size and the large volume and luminosity range spanned, binning would result in a loss of information. Thus, we decided to apply the maximum-likelihood (ML) algorithm first introduced by Marshall et al. (1983) and used recently by Wall et al. (2008) and Ajello et al. (2009a) for the study of (respectively) submillimeter galaxies and blazars detected by Swift. The aim of this analysis is to determine the space density of FSRQs as a function of rest-frame $0.1-100 \mathrm{GeV}$ luminosity $\left(L_{\gamma}\right)$, redshift $(z)$, and photon index $(\Gamma)$, by fitting to the functional form

$\frac{d^{3} N}{d L_{\gamma} d z d \Gamma}=\frac{d^{2} N}{d L_{\gamma} d V} \times \frac{d N}{d \Gamma} \times \frac{d V}{d z}=\Phi\left(L_{\gamma}, z\right) \times \frac{d N}{d \Gamma} \times \frac{d V}{d z}$,

where $\Phi\left(L_{\gamma}, z\right)$ is the LF, and $d V / d z$ is the co-moving volume element per unit redshift and unit solid angle (see, e.g., Hogg 1999). The function $d N / d \Gamma$ is the (intrinsic) photon index distribution and is assumed to be independent of $z$. It is modeled as a Gaussian:

$$
\frac{d N}{d \Gamma}=e^{-\frac{(\Gamma-\mu)^{2}}{2 \sigma^{2}}}
$$

where $\mu$ and $\sigma$ are, respectively, the mean and the dispersion of the Gaussian distribution. The spectrum of most blazars is known to deviate from a simple power law (see, e.g., Abdo et al. 2010d, and Section 5). However, detection of sources in Fermi is performed by means of an ML fit (to the source and background photons) by modeling the source spectrum with a power law (Abdo et al. 2010a). For consistency, we follow here the same procedure and assess in the Appendix the uncertainties connected with this hypothesis.

The best-fit LF is found by comparing, through an ML estimator, the number of expected objects (for a given model LF) to the observed number while accounting for selection effects in the survey. In this method, the space of luminosity, redshift, and photon index is divided into small intervals of size $d L_{\gamma} d z d \Gamma$. In 
each element, the expected number of blazars with luminosity $L_{\gamma}$, redshift $z$, and photon index $\Gamma$ is

$$
\begin{aligned}
\lambda\left(L_{\gamma}, z, \Gamma\right) d L_{\gamma} d z d \Gamma= & \Phi\left(L_{\gamma}, z\right) \Omega\left(L_{\gamma}, z, \Gamma\right) \\
& \times \frac{d N}{d \Gamma} \frac{d V}{d z} d L_{\gamma} d z d \Gamma,
\end{aligned}
$$

where $\Omega\left(L_{\gamma}, z, \Gamma\right)$ is the sky coverage and represents the probability of detecting in this survey a blazar with luminosity $L_{\gamma}$, redshift $z$, and photon index $\Gamma$. This probability was derived for the sample used here by Abdo et al. (2010f) and the reader is referred to that aforementioned paper for more details. With sufficiently fine sampling of the $L_{\gamma}-z-\Gamma$ space the infinitesimal element will either contain 0 or 1 FSRQs. In this regime one has a likelihood function based on joint Poisson probabilities:

$$
\begin{aligned}
L & =\prod_{i} \lambda\left(L_{\gamma, i}, z_{i}, \Gamma_{i}\right) d L_{\gamma} d z d \Gamma e^{-\lambda\left(L_{\gamma, i}, z_{i}, \Gamma_{i}\right) d L_{\gamma} d z d \Gamma} \\
& \times \prod_{j} e^{-\lambda\left(L_{\gamma, j}, z_{j}, \Gamma_{j}\right) d L_{\Gamma} d z d \Gamma}
\end{aligned}
$$

This is the combined probability of observing one blazar in each bin of $\left(L_{\gamma, i}, z_{i}, \Gamma_{i}\right)$ populated by one Fermi FSRQ and zero FSRQs for all other $\left(L_{\gamma, j}, z_{j}, \Gamma_{j}\right)$. Transforming to the standard expression $B=-2 \ln L$ and dropping terms which are not model dependent, we obtain

$$
\begin{aligned}
B= & -2 \sum_{i} \ln \frac{d^{3} N}{d L_{\gamma} d z d \Gamma} \\
& +2 \int_{\Gamma_{\min }}^{\Gamma_{\max }} \int_{L_{\gamma, \min }}^{L_{\gamma, \max }} \int_{z_{\min }}^{z_{\max }} \lambda\left(L_{\gamma}, \Gamma, z\right) d L_{\gamma} d z d \Gamma
\end{aligned}
$$

The limits of integration of Equation (5), unless otherwise stated, are $L_{\gamma, \min }=10^{44} \mathrm{erg} \mathrm{s}^{-1}, L_{\gamma, \max }=10^{52} \mathrm{erg} \mathrm{s}^{-1}, z_{\min }=$ $10^{-2}, z_{\max }=6, \Gamma_{\min }=1.8$, and $\Gamma_{\max }=3.0$. The results of the analysis do not depend on the limits of integration $z_{\max }$ and $L_{\gamma, \max }$. The values of the $L_{\gamma, \min }$ and $z_{\min }$ are chosen to be a factor of a few lower than the smallest values observed in Ackermann et al. (2011) to force the LF to account for the paucity of low-luminosity low-redshift FSRQs. However, we get results compatible within the statistical uncertainties if we use the minimum observed luminosity and redshift of the source sample of Table 1 . The best-fit parameters are determined by minimizing ${ }^{6} B$ and the associated $1 \sigma$ error is computed by varying the parameter of interest, while the others are allowed to float, until an increment of $\Delta B=1$ is achieved. This gives an estimate of the $68 \%$ confidence region for the parameter of interest (Avni 1976). While computationally intensive, Equation (5) has the advantage that each source has its appropriate individual detection efficiency and $k$-correction treated independently.

In order to test whether the best-fit LF provides a good description of the data we compare the observed redshift, luminosity, index, and source count distributions against the prediction of the LF. The first three distributions can be obtained from the LF as

$$
\frac{d N}{d z}=\int_{\Gamma_{\min }}^{\Gamma_{\max }} \int_{L_{\gamma, \min }}^{L_{\gamma, \max }} \lambda\left(L_{\gamma}, \Gamma, z\right) d L_{\gamma} d \Gamma
$$

\footnotetext{
6 The MINUIT minimization package, embedded in ROOT (root.cern.ch),
} has been used for this purpose.

$$
\begin{gathered}
\frac{d N}{d L_{\gamma}}=\int_{\Gamma_{\min }}^{\Gamma_{\max }} \int_{z_{\min }}^{z_{\max }} \lambda\left(L_{\gamma}, \Gamma, z\right) d z d \Gamma \\
\frac{d N}{d \Gamma}=\int_{L_{\gamma, \min }}^{L_{\gamma, \max }} \int_{z_{\min }}^{z_{\max }} \lambda\left(L_{\gamma}, \Gamma, z\right) d L_{\gamma} d z,
\end{gathered}
$$

where the extremes of integrations are the same as in Equation (5). Since $L_{\gamma}$ depends on redshift, Equations (6) and (7) are not independent. The source count distribution can be derived as

$$
N(>S)=\int_{\Gamma_{\min }}^{\Gamma_{\max }} \int_{z_{\min }}^{z_{\max }} \int_{L_{\gamma}(z, S)}^{L_{\gamma, \max }} \Phi\left(L_{\gamma, z}\right) \frac{d N}{d \Gamma} \frac{d V}{d z} d \Gamma d z d L_{\gamma},
$$

where $L_{\gamma}(z, S)$ is the luminosity of a source at redshift $z$ having a flux of $S$.

To display the LF we rely on the " $N$ obs $/ N^{\text {mdl " }}$ method devised by La Franca \& Cristiani (1997) and Miyaji et al. (2001) and employed in several recent works (e.g., La Franca et al. 2005; Hasinger et al. 2005). Once a best-fit function for the LF has been found, it is possible to determine the value of the observed LF in a given bin of luminosity and redshift:

$$
\Phi\left(L_{\gamma, i}, z_{i}\right)=\Phi^{\mathrm{mdl}}\left(L_{\gamma, i}, z_{i}\right) \frac{N_{i}^{\mathrm{obs}}}{N_{i}^{\mathrm{mdl}}},
$$

where $L_{\gamma, i}$ and $z_{i}$ are the luminosity and redshift of the $i$ th bin, $\Phi^{\mathrm{mdl}}\left(L_{\gamma, i}, z_{i}\right)$ is the best-fit LF model, and $N_{i}^{\text {obs }}$ and $N_{i}^{\mathrm{mdl}}$ are the observed and the predicted number of FSRQs in that bin. These two techniques (the Marshall et al. 1983 ML method and the " $N$ obs $/ N^{\text {mdl } " ~ e s t i m a t o r) ~ p r o v i d e ~ a ~ m i n i m a l l y ~ b i a s e d ~ e s t i m a t e ~ o f ~}$ the LF (cf. Miyaji et al. 2001).

\section{RESULTS}

\subsection{Pure Luminosity Evolution and the Evidence for a Redshift Peak}

The space density of radio-quiet AGNs is known to be maximal at intermediate redshift. The epoch of this "redshift peak" correlates with source luminosity (e.g., Ueda et al. 2003; Hasinger et al. 2005). This peak may represent the combined effect of SMBH growth over cosmic time and a falloff in fueling activity as the rate of major mergers decreases at late times. To test whether such behavior is also typical of the LAT FSRQ population, we perform a fit to the data using a pure luminosity evolution (PLE) model of the form

$$
\Phi\left(L_{\gamma}, z\right)=\Phi\left(L_{\gamma} / e(z)\right)
$$

where

$$
\begin{aligned}
\Phi\left(L_{\gamma} / e(z=0)\right) & =\frac{d N}{d L_{\gamma}} \\
& =\frac{A}{\ln (10) L_{\gamma}}\left[\left(\frac{L_{\gamma}}{L_{*}}\right)^{\gamma_{1}}+\left(\frac{L_{\gamma}}{L_{*}}\right)^{\gamma 2}\right]^{-1}
\end{aligned}
$$

and

$$
e(z)=(1+z)^{k} e^{z / \xi}
$$

In this model, the evolution is entirely in luminosity, i.e., the FSRQ were more luminous in the past if positive evolution $(k>0)$ is found (the opposite is true otherwise). It is also 

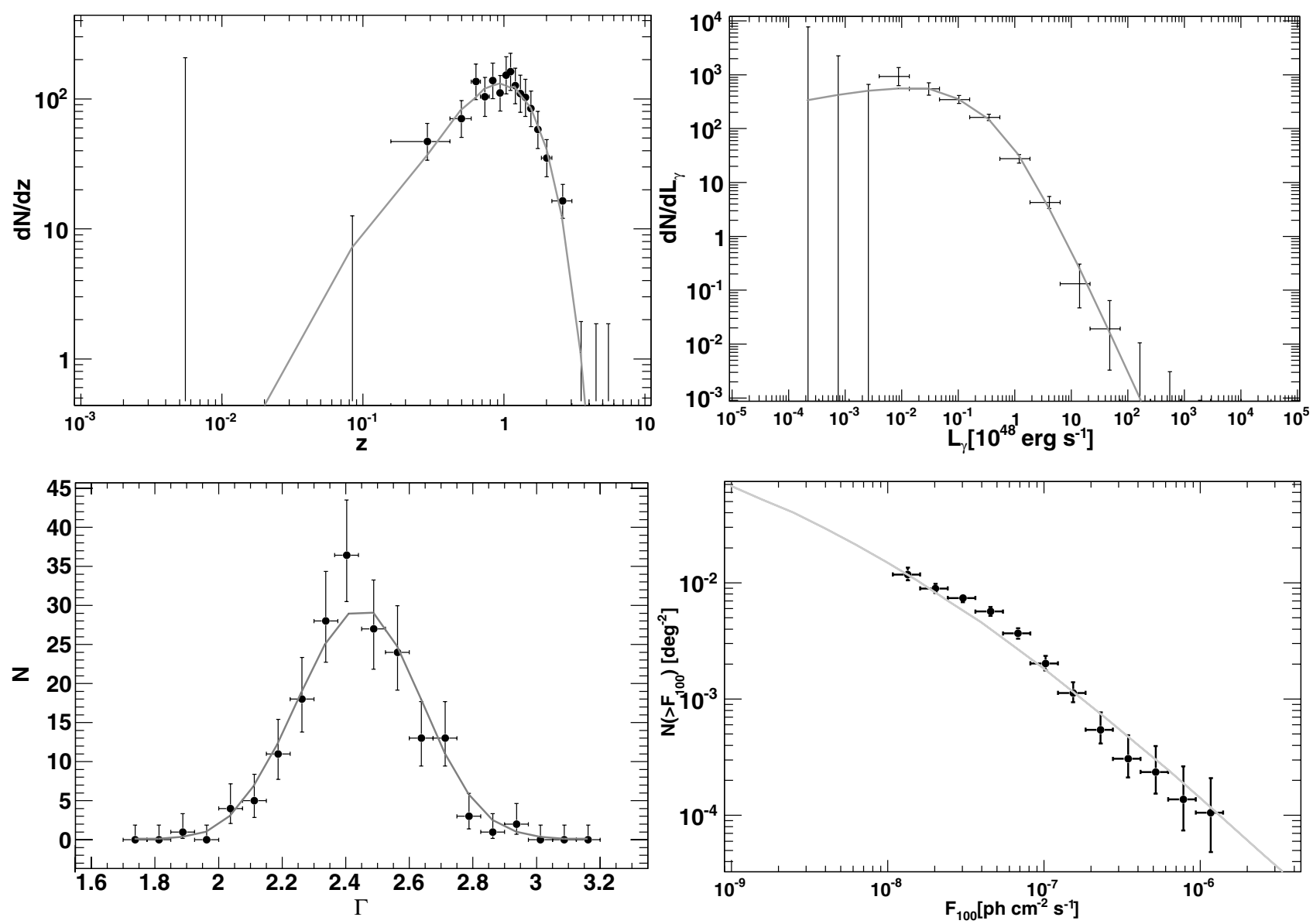

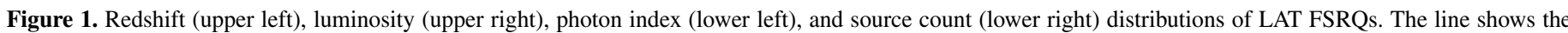
best-fit PLE model convolved with the selection effects of Fermi discussed in the text.

Table 2

Best-fit Parameters of the Pure Luminosity Evolution LF

\begin{tabular}{|c|c|c|c|c|c|c|c|c|c|}
\hline Sample & No. of Objects & $A^{\mathrm{a}}$ & $\gamma_{1}$ & $L_{*}$ & $\gamma_{2}$ & $\mathrm{k}$ & $\xi$ & $\mu$ & $\sigma$ \\
\hline ALL & 186 & $5.59( \pm 0.41) \times 10^{3}$ & $0.29 \pm 0.53$ & $0.026 \pm 0.066$ & $1.25 \pm 0.32$ & $5.70 \pm 1.02$ & $-0.46 \pm 0.01$ & $2.45 \pm 0.13$ & $0.18 \pm 0.01$ \\
\hline Low L & 89 & $15.4( \pm 0.2) \times 10^{3}$ & 0.29 & 0.026 & 1.25 & $4.30 \pm 2.39$ & $-0.50 \pm 0.04$ & $2.47 \pm 0.04$ & $0.21 \pm 0.03$ \\
\hline High L & 97 & $22.6( \pm 2.0) \times 10^{3}$ & 0.29 & 0.026 & 1.25 & $3.47 \pm 1.73$ & $-0.79 \pm 0.04$ & $2.46 \pm 0.02$ & $0.20 \pm 0.02$ \\
\hline
\end{tabular}

Notes. Parameters without an error estimate were kept fixed during the fit.

${ }^{\mathrm{a}}$ In units of $10^{-13} \mathrm{Mpc}^{-3} \mathrm{erg}^{-1} \mathrm{~s}$.

straightforward to demonstrate that the luminosity evolution (i.e., Equation (13)) of FSRQs peaks at $z_{c}=-1-k \xi$. The bestfit parameters are reported in Table 2. The evolution of the FSRQ class is found to be positive and fast $(k=5.70 \pm 1.02)$. The redshift peak is $z_{c}=1.62 \pm 0.03$. Moreover, the subsequent rate of decrease of the luminosity after the peak is well constrained $(\xi=-0.46 \pm 0.01)$. However, as shown in Figure 1, while this model provides a good fit to the observed redshift and luminosity distributions, it is a very poor representation of the measured $\log N-\log S$.

We next test whether the redshift peak depends on luminosity, splitting the data set at $L_{\gamma}=3.2 \times 10^{47} \mathrm{erg} \mathrm{s}^{-1}$. A fit is then performed to each sub-sample to determine the position of the redshift peak (if any), keeping the parameters of Equation (12) fixed. The results of the fits to the low- and high-luminosity data sets are reported in Table 2. From Equation (13) and the values of $k$ and $\xi$ it is apparent that there is a significant shift in the redshift peak, with the low- and high-luminosity samples peaking at $\sim 1.15$ and $\sim 1.77$, respectively.

\subsection{The Luminosity-dependent Density Evolution and the Redshift Peak}

Since a simple PLE LF model provides an inadequate fit to the Fermi data and since there is some evidence for the evolution of the redshift peak with luminosity, we now fit the Fermi FSRQ set to a luminosity-dependent density evolution (LDDE) model. Here, the evolution is primarily in density with a luminositydependent redshift peak. The LDDE model is parameterized as

$$
\Phi\left(L_{\gamma}, z\right)=\Phi\left(L_{\gamma}\right) \times e\left(z, L_{\gamma}\right),
$$

where

$$
e\left(z, L_{\gamma}\right)=\left[\left(\frac{1+z}{1+z_{c}\left(L_{\gamma}\right)}\right)^{p 1}+\left(\frac{1+z}{1+z_{c}\left(L_{\gamma}\right)}\right)^{p 2}\right]^{-1}
$$

and

$$
z_{c}\left(L_{\gamma}\right)=z_{c}^{*} \cdot\left(L_{\gamma} / 10^{48}\right)^{\alpha} .
$$



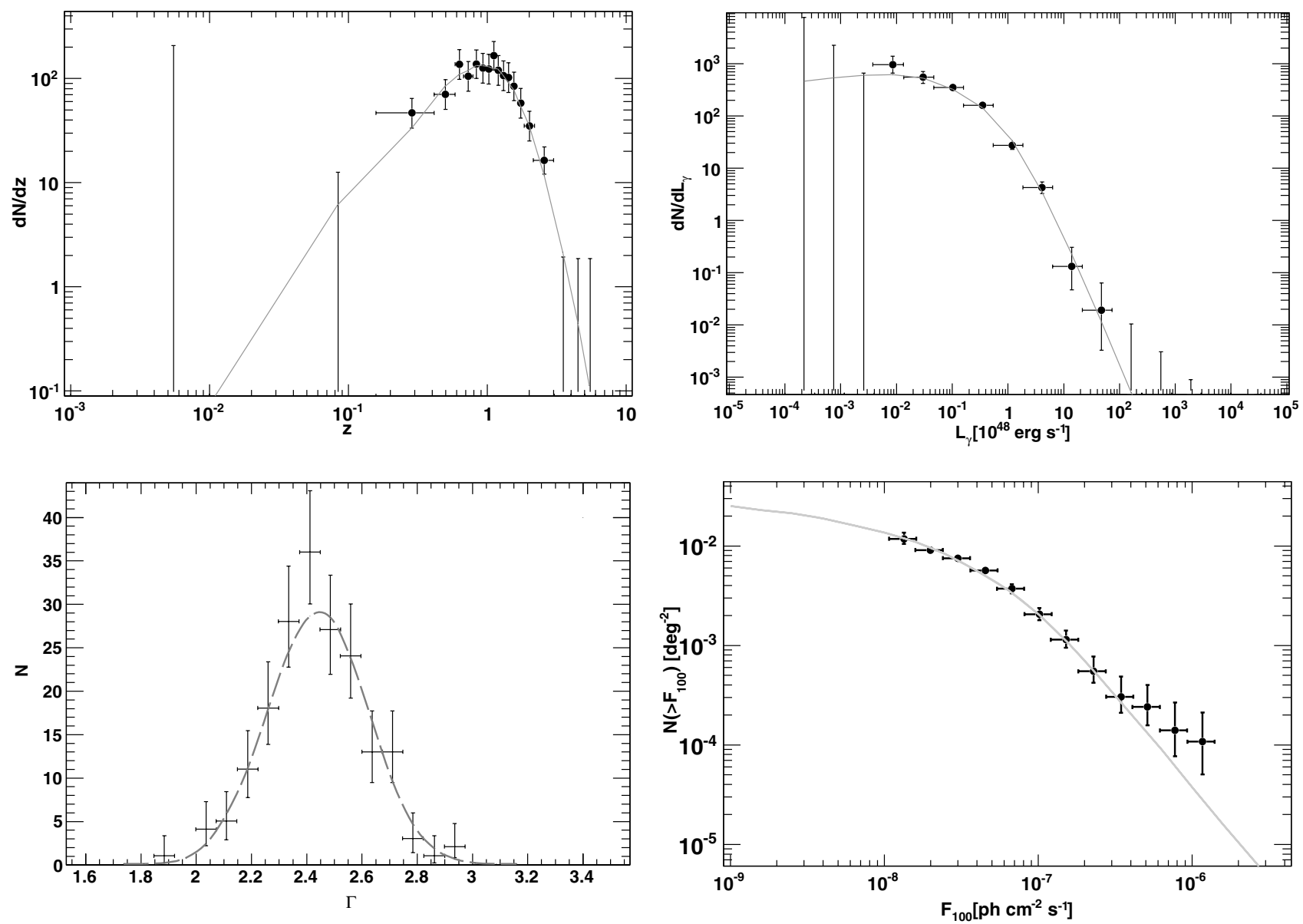

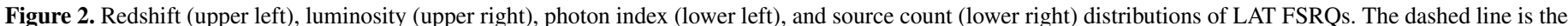
best-fit LDDE model convolved with the selection effects of Fermi. Note the improved source count distribution over the predictions of the PLE model of Figure 1.

$\Phi\left(L_{\gamma}\right)$ is the same double power law used in Equation (12). This parameterization is similar to that proposed by Ueda et al. (2003), but is continuous around the redshift peak $z_{c}\left(L_{\gamma}\right)$. This has obvious advantages for fitting algorithms that rely on the derivatives of the fitting function to find the minimum. Here $z_{c}\left(L_{\gamma}\right)$ corresponds to the (luminosity-dependent) redshift where the evolution changes sign (positive to negative), with $z_{c}^{*}$ being the redshift peak for an FSRQ with a luminosity of $10^{48} \mathrm{erg} \mathrm{s}^{-1}$.

The LDDE model provides a good fit to the LAT data and is able to reproduce the observed distribution in Figure 2. The loglikelihood ratio test shows that the improvement over the best PLE model is significant, with a chance probability of $\sim 10^{-6}$. Results are reported in Table 3.

In Figure 3, we subdivide the sample into four redshift bins with comparable number of sources to illustrate how the LF changes. The evolution, visible as a shifting of the peak and a change of the shape of the LF between different bins of redshift, is clearly visible. This evolution takes place mostly below redshift $\sim 1.1$ where the space density of our least luminous objects (i.e., $L_{\gamma} \approx 10^{46} \mathrm{erg} \mathrm{s}^{-1}$ ) increases by $\sim 10$ times. Above this redshift the variation is less marked, but one notices that

1. the space density of $\log L=47$ objects decreases from redshift 1 to redshift 1.5 while that of $\log L=48$ FSRQs still increases (lower left panel, green versus red lines). This indicates that the space density of $\log L=47$ FSRQs peaks at a redshift $\sim 1.1$; and
2. likewise, the space density of $\log L=48$ FSRQs reaches its maximum well below $z=3$.

The best-fit parameters confirm that the redshift of maximum space density increases with increasing luminosity (with the power-law index of the redshift-peak evolution $\alpha=0.21 \pm 0.03$, see Equation (16)). This redshift evolution can be clearly seen in Figure 4, which shows the change in space density for different luminosity classes.

\subsection{Analysis of Uncertainties}

One of the main uncertainties of our analysis is due to the incompleteness of the FSRQ sample. In Table 1, there are 17 sources with associated radio counterparts lacking optical type and redshift measurements. A fraction of these may be FSRQs. In addition, there are 29 sources without any statistically associated radio counterpart. The lack of radio flux means that these cannot be FSRQ similar to those in the Fermi sample, unless position errors have prevented radio counterpart associations. Thus, even if a few of these sources are mislocalized, ${ }^{7}$ the maximum possible incompleteness of our FSRQ sample is on the order of $20 /(186+20)$, i.e., $\sim 10 \%$.

\footnotetext{
7 Fermi counterpart identification depends on an accurate assessment of uncertainties in the source localization. While the method properly accounts for the catalog uncertainties in the source position, additional systematic uncertainty may be present due to inaccuracies in the PSF model or background model, particularly in regions of bright diffuse Galactic emission. Improved modeling of the PSF or of the diffuse emission may shift the source localization, and hence the counterpart association probabilities, of a few sources.
} 
Table 3

Best-fit Parameters of the Luminosity Dependent Density Evolution LF

\begin{tabular}{|c|c|c|c|c|c|c|c|c|c|c|c|}
\hline Sample & No. of Objects & $A^{\mathrm{a}}$ & $\gamma_{1}$ & $L_{*}$ & $\gamma_{2}$ & $z_{c}^{*}$ & $\alpha$ & $p_{1}$ & $p_{2}$ & $\mu$ & $\sigma$ \\
\hline ALL & 186 & $3.06( \pm 0.23) \times 10^{4}$ & $0.21 \pm 0.12$ & $0.84 \pm 0.49$ & $1.58 \pm 0.27$ & $1.47 \pm 0.16$ & $0.21 \pm 0.03$ & $7.35 \pm 1.74$ & $-6.51 \pm 1.97$ & $2.44 \pm 0.01$ & $0.18 \pm 0.01$ \\
\hline $\mathrm{ALL}^{\mathrm{b}}$ & 208 & $2.82( \pm 0.19) \times 10^{4}$ & $0.26 \pm 0.12$ & $0.87 \pm 0.53$ & $1.60 \pm 0.27$ & $1.42 \pm 0.15$ & $0.20 \pm 0.03$ & $8.21 \pm 1.78$ & $-5.66 \pm 1.73$ & $2.42 \pm 0.01$ & $0.19 \pm 0.01$ \\
\hline$A L L^{c}$ & 186 & $8.72( \pm 0.63) \times 10^{3}$ & $0.38 \pm 0.16$ & $0.89 \pm 0.70$ & $1.60 \pm 0.30$ & $1.38 \pm 0.18$ & $0.18 \pm 0.03$ & $7.71 \pm 1.84$ & $-4.44 \pm 1.78$ & $\ldots$ & $\ldots$ \\
\hline
\end{tabular}

Notes.

a In units of $10^{-13} \mathrm{Mpc}^{-3} \mathrm{erg}^{-1} \mathrm{~s}$.

b 22 unassociated sources were included in this sample by drawing random redshifts from the observed redshift distribution of FSRQs.

${ }^{\mathrm{c}}$ Derived using the detection efficiency for curved reported in Figure 16. 


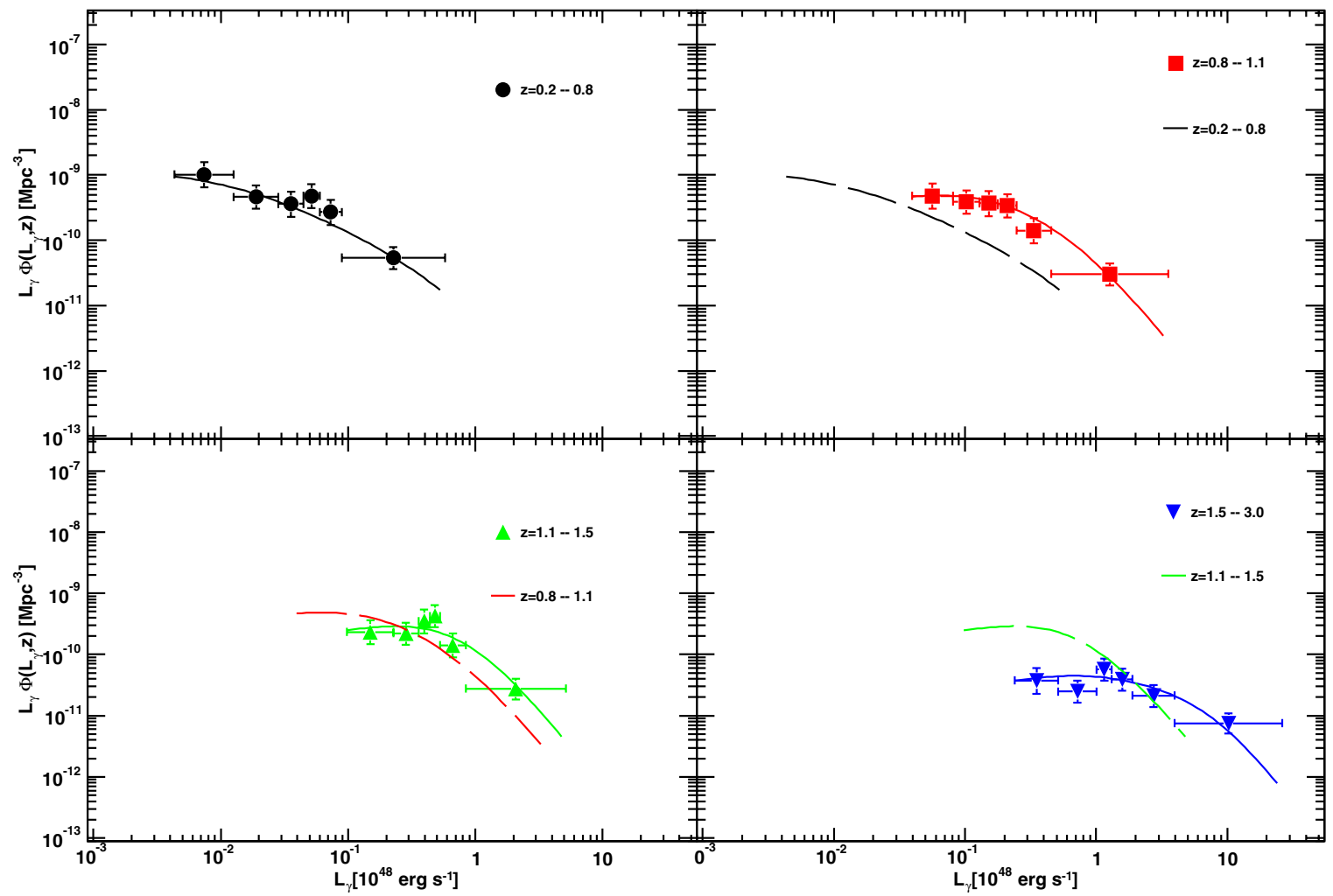

Figure 3. LF of the Fermi FSRQs in different bins of redshift, reconstructed using the $N_{\mathrm{obs}} / N_{\mathrm{mdl}}$ method. The lines represent the best-fit LDDE model of Section 4.2. To highlight the evolution, the LF from the next lower redshift bin is overplotted (dashed lines).

(A color version of this figure is available in the online journal.)

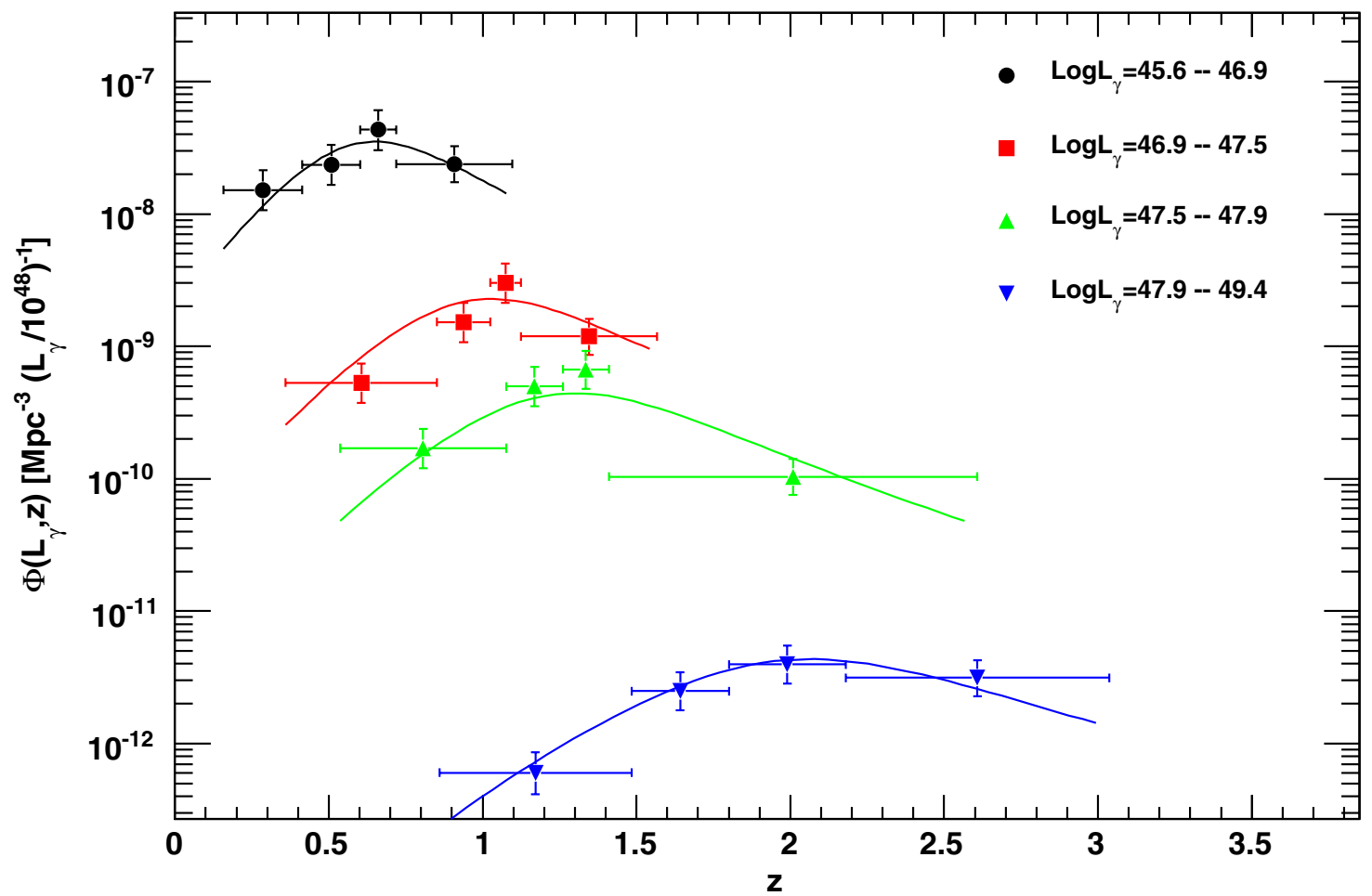

Figure 4. Growth and evolution of different luminosity classes of FSRQs. Note that the space density of the most luminous FSRQs peaks earlier in the history of the universe while the bulk of the population (i.e., the low luminosity objects) are more abundant at later times. The range of measured distribution is determined by requiring at least one source within the volume (lower left) and sensitivity limitations of Fermi (upper right).

(A color version of this figure is available in the online journal.) 
The standard way to account for this incompleteness is to correct upward the normalization of the LF as to reflect the likely real number of FSRQs (associated or not) in our sample. Considering extra information about these sources, this likely incompleteness is even smaller than the $10 \%$ above. First, we find that only $50 \%$ of all the RL identified sources in our sample are FSRQs. This suggests that only 8 of the 17 radio identified sources are FSRQs, with the remainder being BL Lac objects and lower luminosity AGNs. A similar argument can be made based on the $\gamma$-ray spectral index. The median index for FSRQs is $\Gamma=2.44$ while only $9 \%$ of our BL Lac objects have such soft spectra. There are 4 such soft sources in our set of 17 radio sources. Conservatively assuming that all are FSRQs, we infer that twice this number, namely eight, FSRQs are in the radio-detected sample. Of the 29 sources without radio counterparts, four are Blazar "ANTI-Associations" (see Abdo et al. 2009, 2010g, for details), where we can definitively exclude any flat-spectrum radio source bright enough for a Fermi-type blazar. While most of these 29 sources lack the very deep radio observations to make an ANTI-Association, all but five have been classified as pulsar candidates, based on gammaray spectral curvature and lack of variability. We thus suspect that virtually all of these sources are other classes of gamma-ray emitters, e.g., pulsars yet to be discovered, starburst galaxies, etc. Our conclusion is that the likely incompleteness is only $8 /(186+8)=4 \%$. Conservatively adding a few nominally radioquiet sources from erroneous LAT localizations may allow 5\% incompleteness. This correction has been applied in Sections 4.1 and 4.2 .

One might be concerned that the handful of LAT sources that could be unidentified FSRQs occupy an unusual distribution in redshift space and could thus bias the LF. While this is possible, in the present detected set there is no clear redshift trend with radio flux. There is a dearth of blazars at high redshift that are very bright in the optical, but the optically faintest blazars in the set with spectroscopic redshifts happen to have $z<1$. Thus, we can assume that the handful of missing blazars would show a similar redshift distribution to the identified sources. With this assumption, even if 8 of the RL sources are FSRQs and half of the 29 sources without radio counterparts are also FSRQs, we find that the maximum plausible incompleteness of $\sim 22$ (i.e., $8+29 / 2$ ) objects is insufficient to perturb any LF parameter out of the range allowed by the present statistical uncertainty in the fit (see Table 3). Thus incompleteness is not a significant source of uncertainty in our study. In the Appendix, we also argue that other possible sources of systematic uncertainty (detection efficiency, blazar variability, and absorption by extragalactic background light, EBL) do not contribute significantly to the uncertainty in the functional form or the derived parameters of the LF.

\subsection{Comparison with Previous Results}

\subsubsection{The Local Luminosity Function}

The local LF is the luminosity function at redshift 0 . For an evolving population, the local LF is obtained by de-evolving the luminosities (or the densities) according to the best-fit model. This is generally done using the $1 / V_{\mathrm{MAX}}$ method of Schmidt (1968), as reported for example by Della Ceca et al. (2008). However, since the best representation of the LF is the LDDE model, the maximum volume has to be weighted by the density evolution relative to the luminosity of the source. In this case, the maximum allowed volume for a given source is defined as

$$
V_{\mathrm{MAX}}=\int_{z_{\min }}^{z_{\max }} \Omega\left(L_{i}, z\right) \frac{e\left(z, L_{i}\right)}{e\left(z_{\min }, L_{i}\right)} \frac{d V}{d z} d z,
$$

where $L_{i}$ is the source luminosity, $\Omega\left(L_{i}, z\right)$ is the sky coverage, $z_{\max }$ is the redshift above which the source drops out of the survey, and $e\left(z, L_{i}\right)$ is the evolution term of Equation (15) normalized (through $e\left(z_{\min }, L_{i}\right)$ ) at the redshift $z_{\min }$ to which the LF is to be de-evolved. The LF de-evolved at $z_{\min }\left(z_{\min }=0\right.$ in this case) is built using the standard $1 / V_{\text {MAX }}$ method (Schmidt 1968).

In order to gauge the uncertainties that the different methods might introduce in the determination of the local LF we consider also an alternate method. We perform a Monte Carlo simulation, drawing 1000 series of parameters from the covariance matrix of the best-fit LDDE model described in Section 4.2. Using the covariance matrix ensures that parameters are drawn correctly, taking into account their correlations. The re-sampled parameters are used to compute the $\pm 1 \sigma$ error of the LF at redshift 0 . This is reported in Figure 5. There is very good agreement with the local LFs using this method and the $1 / V_{\text {MAX }}$ approach. The gray band in Figure 5 shows the true statistical uncertainty on the space density that the $1 / V_{\text {MAX }}$ method (applied using the best-fit parameters) is not able to capture.

We find a local LF described by a power law with index of 1.6-1.7, for $F_{100}<10^{47} \mathrm{erg} \mathrm{s}^{-1}$, steepening at higher luminosity. Thus, the local LF can be parameterized as a double power law:

$$
\Phi\left(L_{\gamma}\right)=\frac{d N}{d L_{\gamma}}=A\left[\left(\frac{L_{\gamma}}{L_{*}}\right)^{\gamma_{1}}+\left(\frac{L_{\gamma}}{L_{*}}\right)^{\gamma^{2}}\right]^{-1},
$$

where $A=(3.99 \pm 0.30) \times 10^{-11}, L_{*}=0.22 \pm 0.30, \gamma_{1}=$ $1.68 \pm 0.17, \gamma_{2}=3.15 \pm 0.63$, and both $L_{\gamma}$ and $L_{*}$ are in units of $10^{48} \mathrm{erg} \mathrm{s}^{-1}$. Other models (e.g., a Schecter function, a simple power law, etc.) do not generally provide as good of a fit to the data. The values of the low-luminosity index $\gamma_{1}$ and the high-luminosity index $\gamma_{2}$ found here are in good agreement with those of $1.63 \pm 0.16$ and $2.3 \pm 0.3$ reported by Padovani et al. (2007) for the DRBXS survey of FSRQs.

Values very similar to those found here were also reported for a radio FSRQ sample by Dunlop \& Peacock (1990), who find ${ }^{8}$ $\gamma_{1}=1.83$ and $\gamma_{2}=2.96$. The Fermi LF low-luminosity index (i.e., $\left.\gamma_{1}\right)$ is flatter than that determined using EGRET blazars by Narumoto \& Totani (2006) as is apparent in Figure 5. However, a re-analysis of the same data sets employing the blazar sequence (Fossati et al. 1998) to model the blazar SEDs found a lowluminosity index in the 1.8-2.1 range (Inoue \& Totani 2009). Also, in a more recent work, Inoue et al. (2010) modified their SED models to be able to reproduce TeV data of known blazars. Their LF at redshift 0 (see Figure 5) is found to be in relatively good agreement with that found here for the Fermi sample.

\subsubsection{The Luminosity Function at Redshift 1}

Figure 6 shows the luminosity of FSRQ at redshift 1 compared to predictions from recent models. It is apparent that the evolution of the Fermi LF is stronger than predicted by any of these models. The increase in space density from redshift 0

\footnotetext{
8 Their definition of local luminosity function and Equation (18) differ by a $1 / L_{\gamma}$ (or $1 / \mathrm{P}$ in their paper) term. Thus, we added 1.0 to their exponents.
} 


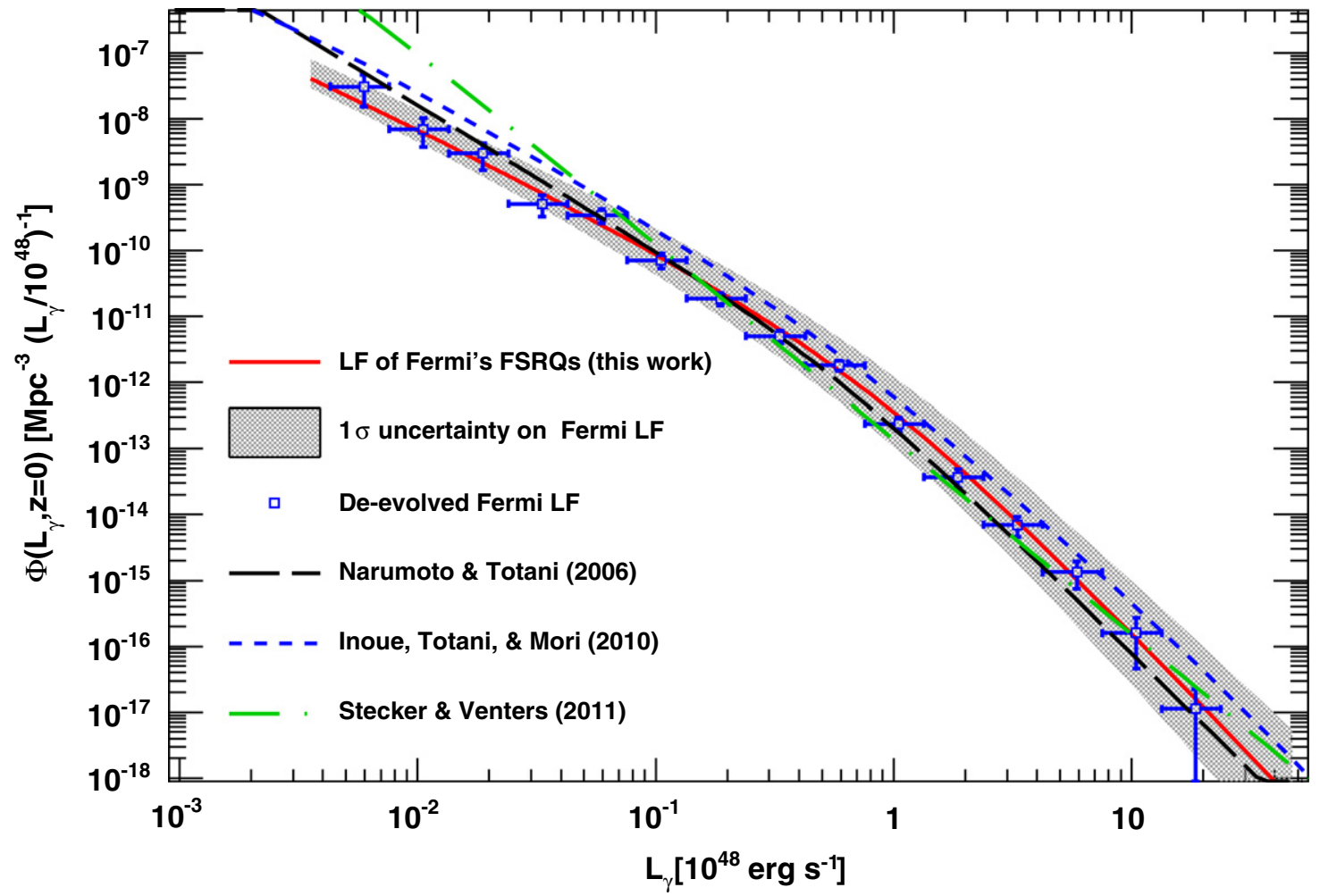

Figure 5. Local $(z=0)$ LF of the Fermi FSRQs as derived from the best-fit LDDE model in Section 4.2 (solid line). The gray band represents the $\pm 1 \sigma$ uncertainty computed as described in the text. The long- and short-dashed lines show the LF models based on the EGRET blazars derived by Narumoto $\&$ Totani (2006) and Inoue et al. (2010), respectively. The dash-dotted line shows the prediction from the model of Stecker \& Venters (2011).

(A color version of this figure is available in the online journal.)

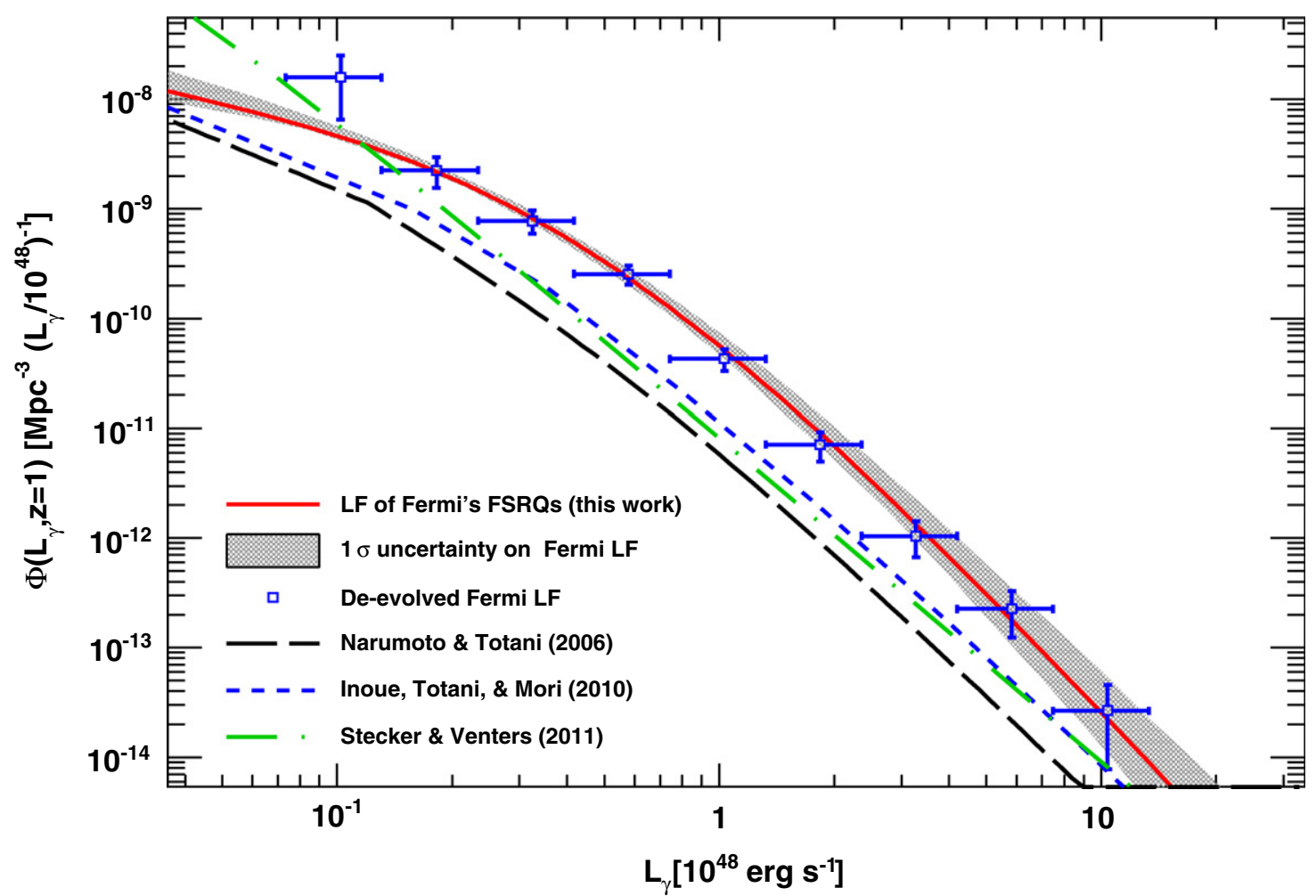

Figure 6. LF of the Fermi FSRQs at redshift 1.0 as derived from the best-fit LDDE model in Section 4.2 (solid line). The gray band represents the $\pm 1 \sigma$ uncertainty computed with the method described in the text. The long-dashed and short-dashed lines show the LF models based on the EGRET blazars derived, respectively, by Narumoto \& Totani (2006) and Inoue et al. (2010). The dash-dotted line shows the prediction from the model of Stecker \& Venters (2011).

(A color version of this figure is available in the online journal.) 
to 1 for a source with a luminosity of $10^{48} \mathrm{erg} \mathrm{s}^{-1}$ is almost a factor $\sim 150$. This dramatic increase is not seen in the evolution of radio-quiet AGNs (e.g., Ueda et al. 2003; Hasinger et al. 2005 ) whose space density increases by a factor $25-50$ between redshifts 0 and 1 . The increase of a factor $\sim 60$ seen in FSRQs detected in radio (Dunlop \& Peacock 1990) is still slower than that of Fermi blazars. This explains why the predictions based on LFs derived at other wavelengths (see Figure 6) underpredict the density of high-luminosity Fermi FSRQs at redshift of 1.

\section{THE SPECTRAL ENERGY DISTRIBUTIONS OF FSRQs}

We may use the 0.1-300 GeV LAT spectra of our uniform bright Fermi FSRQ sample along with the 15-200 keV spectra measured by the Swift Burst Alert Telescope (BAT) to characterize the high energy inverse Compton (IC) sector of the blazar SED. Indeed, since Fermi-LAT and Swift-BAT have comparable sensitivity in their respective bands ${ }^{9}$ and since the two bands cover the bulk of the IC component, a joint study allows an accurate characterization of the IC spectrum and the contribution of FSRQs to the cosmic high-energy background. In the next sections, we describe how the data analysis for LAT and BAT was performed.

\subsection{Data Analysis}

Our goal is to produce SEDs for the bright Fermi FSRQs that cover the energy range $15 \mathrm{keV}-300 \mathrm{GeV}$ by combining data from Swift/BAT and Fermi/LAT. For this analysis, we further restrict the sample of Fermi's FSRQs to $F_{100} \geqslant 7 \times$ $10^{-8}$ photons $\mathrm{cm}^{-2} \mathrm{~s}^{-1}$ (corresponding to an energy flux of $3.4 \times 10^{-11} \mathrm{erg} \mathrm{s}^{-1} \mathrm{~cm}^{-2}$ for a power law with an index of 2.45), since for brighter sources Fermi has a negligible bias in the detected spectral indices and FSRQs are selected uniformly (Abdo et al. 2010f). For fainter sources hard-spectrum objects (principally BL Lac objects) dominate the sample. There are 103 bright FSRQs detected by Fermi that meet these criteria (Abdo et al. 2010g). In this section, we describe how the processing of the Fermi data and that of Swift data has been performed.

We analyze two years of Fermi data using version V9r21 of the science tools. ${ }^{10}$ The data are filtered, removing time periods in which the instrument was not in sky-survey mode and photons whose zenith angle is larger than $100^{\circ}$. We consider only photons collected within $15^{\circ}$ of the source position with $100 \mathrm{MeV} \leqslant E \leqslant 300 \mathrm{GeV}$. We employ the P7SOURCE_V6 instrumental response function and perform binned likelihood analysis using the gtlike tool. First, a likelihood fit using a power-law model for all the sources in the region of interest is performed on the entire energy band (100 MeV-300 GeV). The parameters (i.e., flux and photon index) of all the sources within $3^{\circ}$ of the target FSRQ, along with the normalization of the diffuse model, are left free. More distant sources have parameters frozen at the 2FGL measured values (Nolan et al. 2012). We next choose 30 logarithmically spaced energy bins and perform a binned likelihood in each, deriving the flux of the target FSRQ in each energy bin. During this exercise the flux of the FSRQ is allowed to vary while the photon index is fixed at the best-fit value found for the whole band. All the neighboring sources had parameters fixed at the best-fit values, although

\footnotetext{
9 A Fermi FSRQ with a photon flux of $3 \times 10^{-8}$ photons $\mathrm{cm}^{-2} \mathrm{~s}^{-1}$ in the $100 \mathrm{MeV}-100 \mathrm{GeV}$ band and a power-law spectrum with an index of 2.4 has an energy flux of $1.5 \times 10^{-11} \mathrm{erg} \mathrm{cm}^{-2} \mathrm{~s}^{-1}$.

10 http://fermi.gsfc.nasa.gov/ssc/data/analysis/software/
}

the diffuse emission normalization was allowed to vary. This analysis provides a 30 bin $100 \mathrm{MeV}-300 \mathrm{GeV}$ energy spectrum for all 103 sources in the bright FSRQ sample.

Swift-BAT is a coded-mask telescope that has conducted a several-year survey in the 15-200 keV hard X-ray sky (Gehrels et al. 2004; Barthelmy et al. 2005). With this deep exposure, BAT reaches a sensitivity of $\sim 10^{-11} \mathrm{erg} \mathrm{cm}^{-2} \mathrm{~s}^{-1}$ on most of the highlatitude sky (e.g., Tueller et al. 2008; Ajello et al. 2008a, 2008c; Cusumano et al. 2010). Blazars represent $15 \%-20 \%$ of the extragalactic source population detected by BAT (Ajello et al. 2009a). We use $\sim 6$ years of BAT data to extract a $15-200 \mathrm{keV}$ spectrum for all the FSRQs in the Fermi sample. Spectral extraction is performed according to the recipes presented by Ajello et al. (2008c) and discussed in detail by Ajello et al. (2009b) and Ajello et al. (2010).

Both BAT and LAT spectra are multiplied by $4 \pi D_{L}(z)^{2}$ (with $D_{L}(z)$ the luminosity distance at redshift $z$ ) to transform the flux into a luminosity and shifted by $(1+z)$ to transform into source rest-frame SEDs.

For each FSRQ, we fit the BAT and LAT data with an empirical model of the following form:

$$
\begin{aligned}
E^{2} \frac{d N}{d E} \cdot 4 \pi D_{L}(z)^{2}= & E^{2}\left[\left(\frac{E}{E_{b}}\right)^{\gamma_{\mathrm{BAT}}}+\left(\frac{E}{E_{b}}\right)^{\gamma_{\mathrm{LAT}}}\right]^{-1} \\
& \cdot e^{-\sqrt{E / E_{c}}} \cdot 4 \pi D_{L}(z)^{2}
\end{aligned}
$$

where $\gamma_{\text {BAT }}$ and $\gamma_{\text {LAT }}$ are the power-law indices in the BAT and the LAT bands and $E_{b}$ and $E_{c}$ are the break and the cutoff energy, respectively. The $e^{-\sqrt{E / E_{c}}}$ term allows us to model the curvature that is clearly visible in a few of the Fermi spectra. The fit is performed only for $E<20 \mathrm{GeV}$ to avoid possible steeping due to the absorption of $\gamma$-ray photons by the EBL (e.g., Stecker et al. 2006; Franceschini et al. 2008).

Two sample spectra are shown in Figure 7. It is apparent that for the brightest FSRQs, BAT and LAT are efficient in constraining the shape of the IC emission. Even when the BAT signal is not significant, the upper limit from BAT still provides useful constraints on the low energy curvature of the SED. In a number of bright sources (see, e.g., Figure 7) the highestenergy data point in BAT at $\geqslant 120 \mathrm{keV}$ is seen to deviate from the baseline fit. This deviation is at present not significant (i.e., the reduced $\chi^{2}$ of the baseline fit is already $\approx 1.0$ ), but is suggestive of a second component. Observations with INTEGRAL extending to energies $\geqslant 200 \mathrm{keV}$ might ascertain the nature of this feature.

Several caveats necessarily apply to our analysis. First, the BAT and LAT observations are not strictly simultaneous. LAT spectra are accumulated over two years while the BAT data span six years. In principle one could restrict the BAT data to the period spanned by the Fermi observations. In practice this would seriously limit the BAT sensitivity, weakening constraints on most of the spectra. Second, it is possible that BAT and LAT are not sampling exactly the same emission component. In particular, BAT might be dominated by IC emission produced by the synchrotron self-Compton (SSC; Maraschi et al. 1992) component while the LAT may be more sensitive to external Compton (EC; Dermer \& Schlickeiser 1993) emission. Ultimately, detailed SED modeling with strictly simultaneous data would be needed to eliminate these concerns, and such work is well beyond the scope of this paper. Bearing these caveats in mind, our bright sample is nearly free of selection effects other than the hard flux-limit threshold applied 

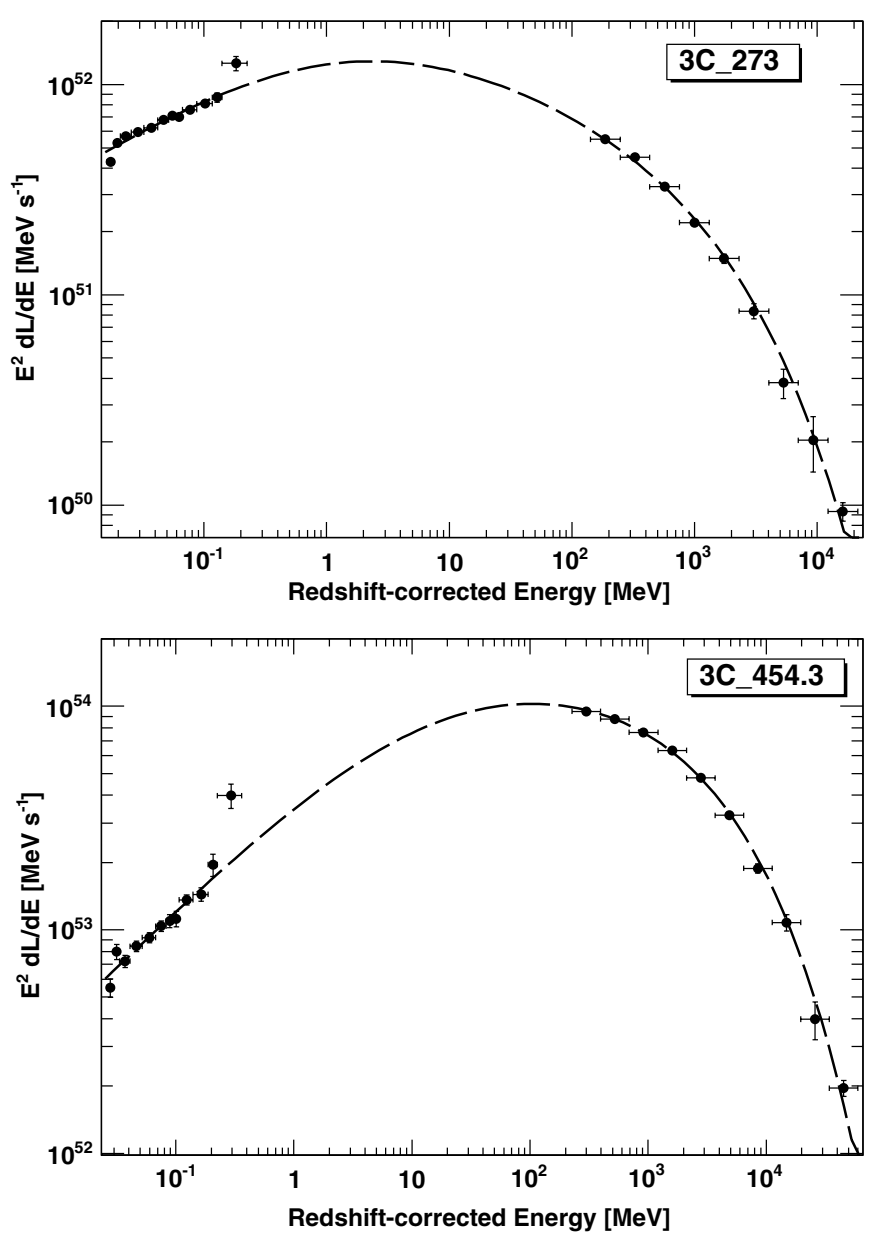

Figure 7. Two BAT-LAT spectra of famous blazars fitted with the empirical model described in Section 5.

to the Fermi data. This allows a detailed study of the average properties of the high-energy SED of FSRQs.

\subsection{Correlation of Peak Luminosity and the Energy of the Peak}

We can compare the IC rest-frame peak luminosity (i.e., the luminosity at the peak of the IC component) and peak energy from the SED fits to the FSRQs in our sample. As shown in Figure 8, there is no apparent correlation between these quantities; indeed the Kendall test gives a value $\tau=0.09$ indicating no significant correlation. Since generally neither Fermi nor BAT directly sample the high-energy peak, we also fit the spectra using a third-degree polynomial function instead of the model in Equation (19). The results are shown in the lower panel of Figure 8 and confirm the previous findings.

This is in contrast to the correlation found (but see also Nieppola et al. 2008) between the luminosity and the energy of the synchrotron peak of blazars (e.g., Ghisellini et al. 1998; Fossati et al. 1998). This might imply that the jet parameters (e.g., Doppler factor, luminosity of the target photon field, etc.) do not depend on blazar GeV luminosity or redshift. This may be understood if the IC peak is largely controlled by EC emission for these sources.

\subsection{Average SEDs}

It is useful to estimate the average SED of FSRQs, particularly for estimating the contribution of FSRQs to the extragalactic gamma-ray background. First we define the bolometric
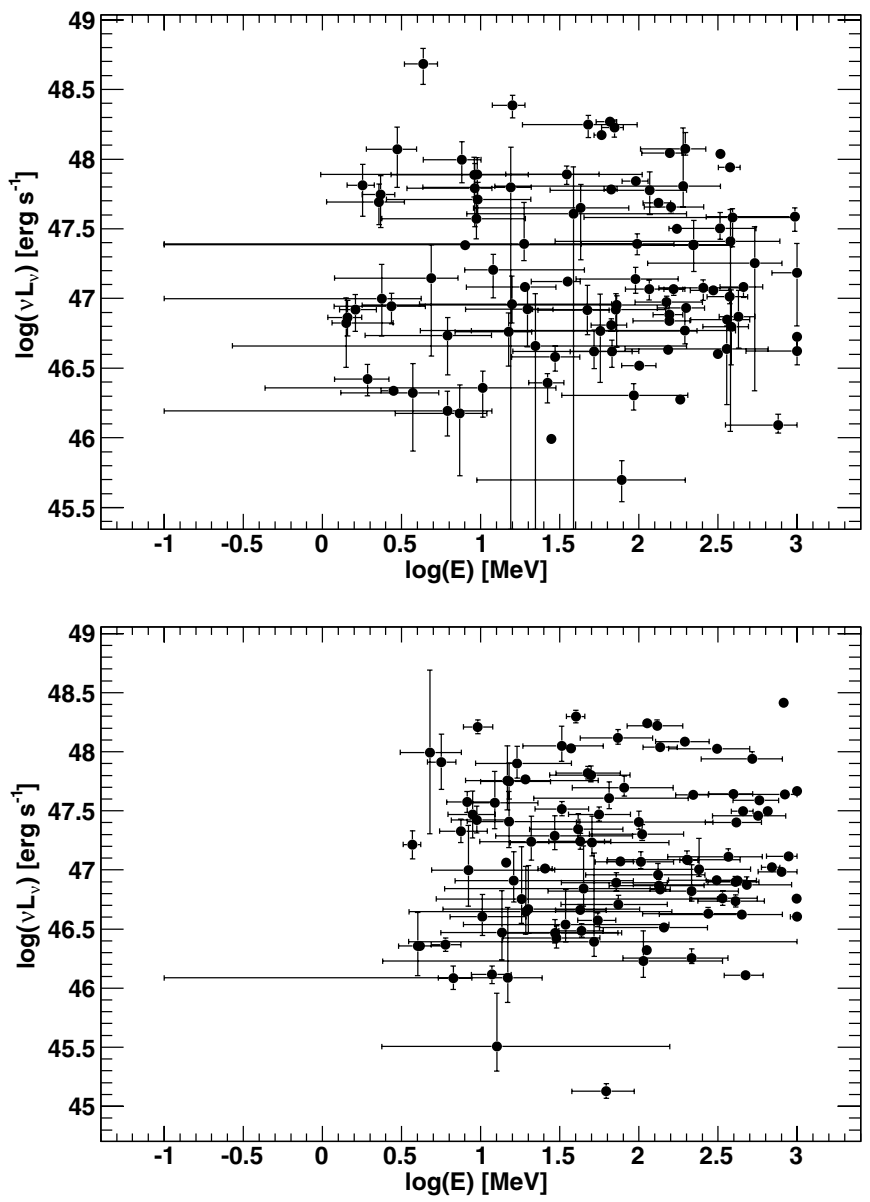

Figure 8. Peak luminosity vs. the energy of the peak for the complete sample of FSRQs discussed in Section 5. The upper plot shows the value derived using a double power law with exponential cutoff while the lower panel shows parameters derived using a third-degree polynomial function.

luminosity as the luminosity in the $10 \mathrm{keV}-300 \mathrm{GeV}$ band ${ }^{11}$ and divide the sources into four bins of bolometric luminosity with approximately the same number of objects in each bin. In these luminosity bins we compute the average of the logarithm of the spectral luminosity at each energy. Associated errors on this average spectrum are computed using the Jackknife technique. In this framework, we neglect uncertainties due to different level of the energy density of the EBL which would affect mostly the high-energy part of the SED (i.e., $\geqslant 20 \mathrm{GeV}$ ).

Figure 9 shows the average rest-frame SED for the FSRQ sample in the four luminosity bins. This plot confirms the lack of a systematic correlation of the peak luminosity and energy. Indeed, all the averaged SEDs show a peak in the $10-100 \mathrm{MeV}$ band and their shape does not change much with luminosity.

To transform luminosities between observed and rest-frame we need the $k$-correction, along with its redshift variation, shown in Figure 10. In practice, there is little difference between the $k$-correction for the average SED computed here (even applying EBL absorption, e.g., Franceschini et al. 2008) and one computed for a power law (i.e., $\left.(1+z)^{\Gamma-2}\right)$ with a photon index of 2.4. Only at large redshifts do the two $k$-corrections start to differ; this difference is only $\sim 5 \%$ at a redshift of 4 . We find that using a power-law index of 2.37 and taking into account EBL absorption allow us to reproduce correctly the $k$-correction up to redshift $\sim 6$.

$\overline{11}$ The best fit is extrapolated from $20 \mathrm{GeV}$ to $300 \mathrm{GeV}$. 


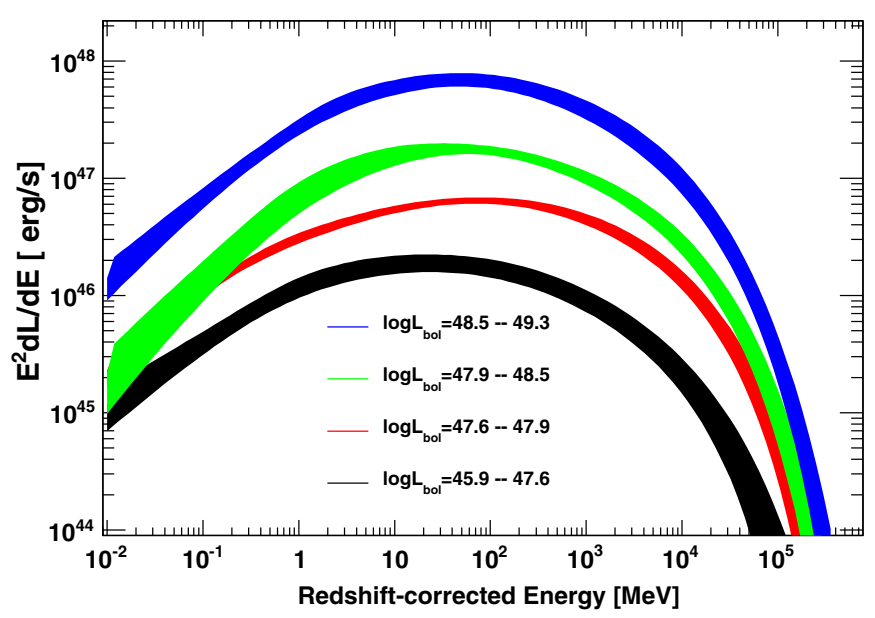

Figure 9. Average rest-frame spectral energy distributions for four representative FSRQ luminosity classes; see Section 5.3. In each SED, the band represents the $1 \sigma$ uncertainty on the average. This does not reflect the uncertainty connected with the level of the extragalactic background light.

(A color version of this figure is available in the online journal.)

\section{THE CONTRIBUTION TO THE ISOTROPIC GAMMA-RAY BACKGROUND}

The nature of the diffuse gamma-ray background at $\mathrm{GeV}$ energies remains one of the most interesting problems in astrophysics. The presence of an isotropic component was first indicated by the OSO-3 satellite (Kraushaar et al. 1972) and confirmed by SAS-2 and EGRET (respectively Fichtel et al. 1975; Sreekumar et al. 1998). This isotropic component is normally referred to as the IGRB. Fermi recently provided a refined measurement of this isotropic component showing that it can be adequately described as a single power law with an index of 2.4 in the $200 \mathrm{MeV}-100 \mathrm{GeV}$ energy range (Abdo et al. 2010e).

Blazars, representing the most numerous identified source population in the EGRET (Hartman et al. 1999) and Fermi (Ackermann et al. 2011) catalogs, are expected to produce a substantial fraction of the IGRB. Typical predictions range from 20\% to 30\% (Chiang \& Mukherjee 1998; Mücke \& Pohl 2000; Narumoto \& Totani 2006; Dermer 2007; Inoue \& Totani 2009 ) to $100 \%$ (Stecker \& Salamon 1996; Stecker \& Venters 2011). Analysis of the source-count distribution showed that for $F_{100} \geqslant 10^{-9}$ photons $\mathrm{cm}^{-2} \mathrm{~s}^{-1}$ the contribution of unresolved blazars to the IGRB is $\sim 16 \%$ in the $100 \mathrm{MeV}-100 \mathrm{GeV}$ band (Abdo et al. 2010f). Since the source count distributions show a strong break at a flux of $F_{100} \approx 6 \times 10^{-8}$ photons $\mathrm{cm}^{-2} \mathrm{~s}^{-1}$, it was concluded that extrapolating the source counts to zero flux would produce $\sim 23 \%$ of the IGRB.

Now, with a measured LF we can more robustly evaluate the emission arising from faint FSRQs. In addition, the FSRQ SED shape study of the previous section also allows improvement over the simple power-law type spectra assumed by Abdo et al. (2010f).

The contribution of "unresolved" FSRQs to the IGRB can be estimated as

$$
\begin{aligned}
F_{\mathrm{EGB}}= & \int_{z_{\min }}^{z_{\max }} d z \frac{d V}{d z} \int_{\Gamma_{\min }}^{\Gamma_{\max }} d \Gamma \int_{L_{\gamma, \min }}^{L_{\gamma, \max }} d L_{\gamma} F_{\gamma}\left(L_{\gamma}, z\right) \\
& \times \frac{d^{3} N}{d L_{\gamma} d z d \Gamma}\left(1-\frac{\Omega\left(\Gamma, F_{\gamma}\right)}{\Omega_{\max }}\right),
\end{aligned}
$$

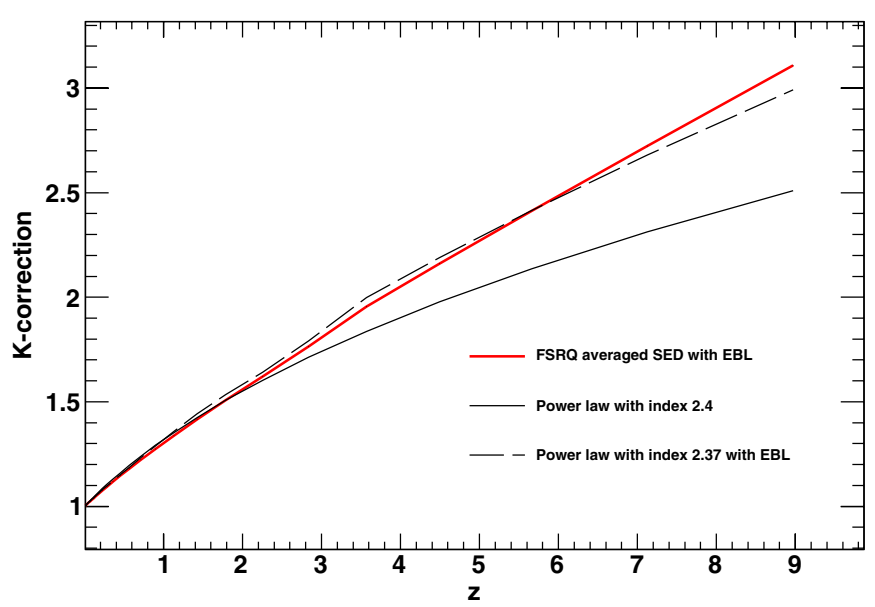

Figure 10. Ratio of source rest-frame luminosity to observed luminosity (i.e., $k$-correction) as a function of redshift for the average SED shape reported in Section 5.3 and for two generic power laws.

(A color version of this figure is available in the online journal.)

where the limits of integration are the same as those of Equation (5) and $F_{\gamma}\left(L_{\gamma}, z\right)$ is the flux of a source with rest-frame luminosity $L_{\gamma}$ at redshift $z$. Since we are interested in the diffuse flux not yet resolved by Fermi (Abdo et al. 2010e) the term $\left(1-\Omega\left(\Gamma, F_{\gamma}\right) / \Omega_{\max }\right)$ takes into account the photon index and source flux dependence of the LAT source detection threshold (see Abdo et al. 2010f for more details).

In Equation (20) setting $\Omega\left(\Gamma, F_{\gamma}\right) / \Omega_{\max }=0$ allows us to compute the total $\gamma$-ray emission arising from the FSRQ class. The result is $3.13_{-0.25}^{+0.37} \times 10^{-6}$ photons $\mathrm{cm}^{-2} \mathrm{~s}^{-1} \mathrm{sr}^{-1}$ in the $100 \mathrm{MeV}-100 \mathrm{GeV}$ band. This value should be compared with the total sky intensity 12 of $\sim 1.4 \times 10^{-5}$ photons $\mathrm{cm}^{-2} \mathrm{~s}^{-1} \mathrm{sr}^{-1}$, which includes IGRB plus detected sources ${ }^{13}$ (Abdo et al. 2010e). Thus, FSRQs make $21.7_{-1.7}^{+2.5} \%$ of this total intensity.

If one considers the contribution only from the FSRQs that Fermi has not detected ${ }^{14}$ (i.e., unresolved sources) then this becomes $9.66_{-1.09}^{+1.67} \times 10^{-7}$ photons $\mathrm{cm}^{-2} \mathrm{~s}^{-1} \mathrm{sr}^{-1}$ (with a maximum systematic uncertainty of $3 \times 10^{-7}$ photons $\mathrm{cm}^{-2} \mathrm{~s}^{-1} \mathrm{sr}^{-1}$; see the Appendix). This represents $9.3_{-1.0}^{+1.6} \%$ of the IGRB intensity in the $0.1-100 \mathrm{GeV}$ band (Abdo et al. 2010e). From above it is also clear that Fermi has already resolved more than 50\% of the total flux arising from the FSRQ class. Figure 11 shows this contribution. The possible presence of EC components in the SEDs of FSRQs makes the estimate between $200 \mathrm{keV}$ and $100 \mathrm{MeV}$ uncertain (see Section 5.3). Future observations with both Fermi above $20 \mathrm{MeV}$ and INTEGRAL above $200 \mathrm{keV}$ and physical modeling of blazar spectra might substantially reduce this uncertainty.

Even the (disfavored) PLE model cannot accommodate a much larger contribution of FSRQs to the IGRB. Indeed, in this case the contribution of unresolved FSRQs would be $1.37 \times 10^{-6}$ photons $\mathrm{cm}^{-2} \mathrm{~s}^{-1} \mathrm{sr}^{-1}$ (or $\sim 13 \%$ of the IGRB intensity). In any case, the sharp falloff of the FSRQ contribution at high and low energies (Figure 11) requires that other sources,

\footnotetext{
12 This does not include the Galactic diffuse emission.

13 This flux is obtained summing the IGRB and the resolved-source fluxes provided in Table 1 of Abdo et al. (2010e) and extrapolating their sum to $E \geqslant 100 \mathrm{MeV}$.

14 This is important for the comparison of the unresolved emission of FSRQs with the measurement of the IGRB which does not include point sources detected by Fermi during the first $\sim 9$ months of observations (Abdo et al. 2010e).
} 

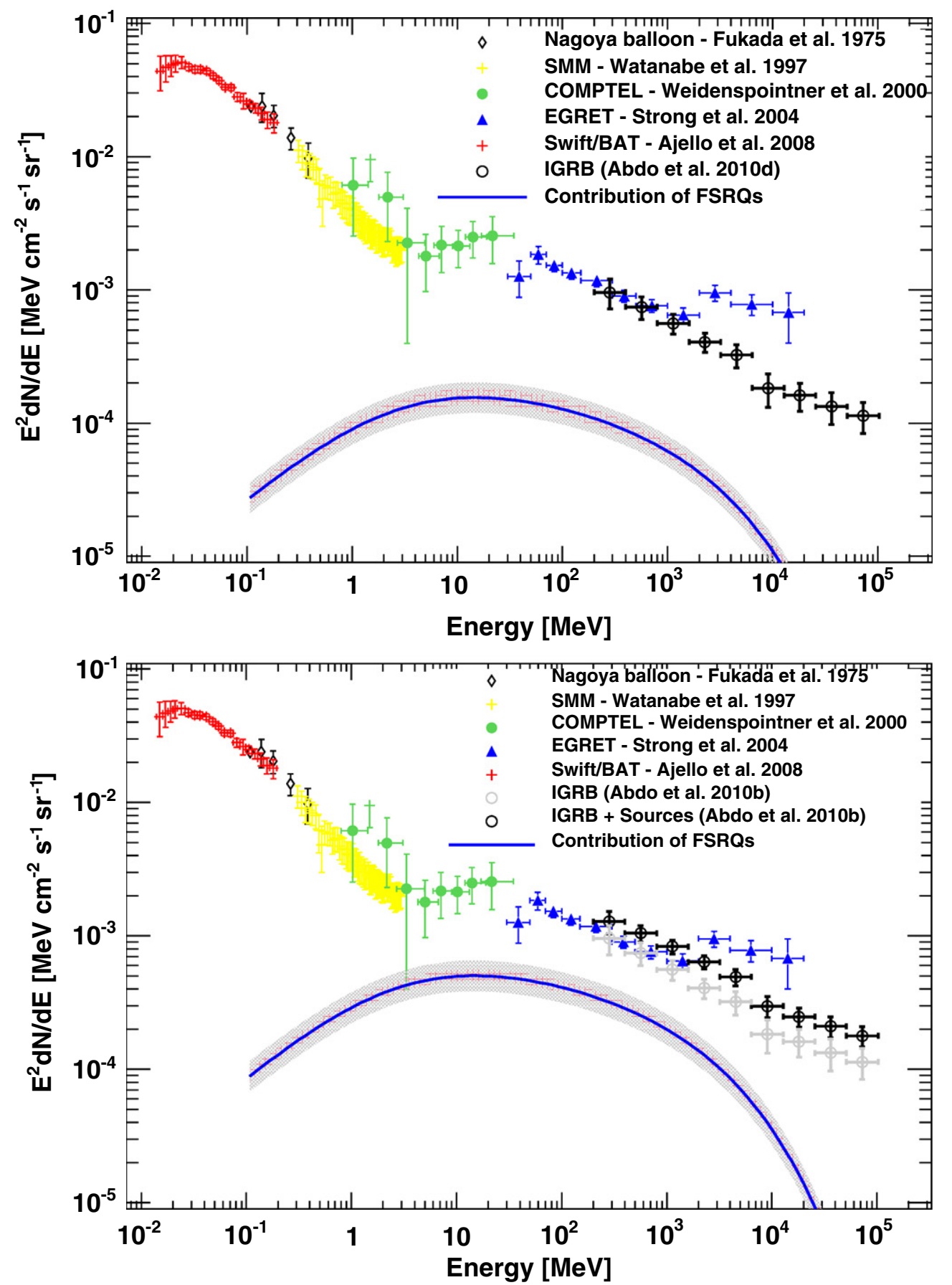

Figure 11. Contribution of unresolved (top) and total (resolved plus unresolved, bottom) FSRQs to the diffuse extragalactic background (blue line) as determined by integrating the luminosity function coupled to the SED model derived in Section 5.3. The hatched band around the best-fit prediction shows the $1 \sigma$ statistical uncertainty while the gray band represents the systematic uncertainty.

(A color version of this figure is available in the online journal.)

e.g., BL Lac objects and starburst galaxies make significant contributions to the IGRB intensity.

\section{BEAMING: THE INTRINSIC LUMINOSITY FUNCTION AND THE PARENT POPULATION}

The luminosities $L$ defined in this work are apparent isotropic luminosities. Since the jet material is moving at relativistic speed $(\gamma>1)$, the observed, Doppler boosted, luminosities are related to the intrinsic values by

$$
L=\delta^{p} \mathscr{L}
$$

where $\mathscr{L}$ is the intrinsic (unbeamed) luminosity and $\delta$ is the kinematic Doppler factor

$$
\delta=\left(\gamma-\sqrt{\gamma^{2}-1} \cos \theta\right)^{-1},
$$

where $\gamma=\left(1-\beta^{2}\right)^{-1 / 2}$ is the Lorentz factor and $\beta=v / c$ is the velocity of the emitting plasma. Assuming that the sources have a Lorentz factor $\gamma$ in the $\gamma_{1} \leqslant \gamma \leqslant \gamma_{2}$ range then the minimum Doppler factor is $\delta_{\min }=\gamma_{2}^{-1}$ (when $\theta=90^{\circ}$ ) and the maximum is $\delta_{\max }=\gamma_{2}+\sqrt{\gamma_{2}^{2}-1}\left(\right.$ when $\theta=0^{\circ}$ ). We adopt a value of $p=4$ that applies to the case of jet emission from a relativistic blob 
radiating isotropically in the fluid frame. The case $p=4$ applies also to spherical blobs if the observed emission is dominated by the SSC component, while a value of $p=5-6$ should be adopted if the emission is due to EC (Dermer 1995). However, as shown later these values imply extremely small isotropic rest-frame luminosities.

Beaming is known to alter the shape of the intrinsic LF. Urry \& Shafer (1984) provide an analytic solution to the case where the intrinsic LF is a single power law and the jets have a single Lorentz factor. In Urry \& Padovani (1991) the intrinsic LF may be a double power law and a distribution of Lorentz factor is considered.

In this section, we will determine the intrinsic LF of the Fermi FSRQs and their Lorentz and Doppler factor distributions. In what follows we adopt the formalism and symbols of Lister (2003) and Cara \& Lister (2008).

We begin by defining the intrinsic LF as

$$
\Phi(\mathscr{L})=k_{1} \mathscr{L}^{-B}
$$

valid in the $\mathscr{L}_{1} \leqslant \mathscr{L} \leqslant \mathscr{L}_{2}$ range. The probability of observing a beamed luminosity $L$ given a Doppler factor $\delta$ is (see also Lister 2003)

$$
P(L, \delta)=P_{\delta}(\delta) * \Phi(\mathscr{L}) \frac{d \mathscr{L}}{d L}
$$

where $P_{\delta}(\delta)$ is the probability density for the Doppler $\delta$. Assuming a random distribution for the jet angles (i.e., $P_{\theta}=$ $\sin \theta$ ), this results in

$$
P_{\delta}(\delta)=\int P_{\gamma}(\gamma) P_{\theta}(\theta)\left|\frac{d \theta}{d \delta}\right| d \gamma=\int P_{\gamma}(\gamma) \frac{1}{\gamma \delta^{2} \beta} d \gamma .
$$

From here it follows that

$$
P_{\delta}(\delta)=\delta^{-2} \int_{f(\delta)}^{\gamma_{2}} \frac{P_{\gamma}(\gamma)}{\sqrt{\gamma^{2}-1}} d \gamma,
$$

where $P_{\gamma}(\gamma)$ is the probability density for $\gamma$ and the lower limit of integration $f(\delta)$ depends on the Doppler factor value and is reported in Equation (A6) in Lister (2003). Integrating over $\delta$ yields the observed LF of the Doppler beamed FSRQs:

$$
\Phi(L)=k_{1} L^{-B} \int_{\delta_{1}(L)}^{\delta_{2}(L)} P_{\delta}(\delta) \delta^{p(B-1)},
$$

where, as in Cara \& Lister (2008), the limits of integration are

$$
\begin{aligned}
& \delta_{1}(L)=\min \left\{\delta_{\max }, \max \left(\delta_{\min },\left(L / \mathscr{L}_{2}\right)^{1 / p}\right)\right\} \\
& \delta_{2}(L)=\max \left\{\delta_{\min }, \min \left(\delta_{\max },\left(L / \mathscr{L}_{1}\right)^{1 / p}\right)\right\} .
\end{aligned}
$$

In this way, by fitting Equation (27) to the Fermi Doppler boosted $\mathrm{LF}$, it is possible to determine the parameters of the intrinsic LF and of the Lorentz-factor distribution.

We assume that the probability density distribution for $\gamma$ is a power law of the form

$$
P_{\gamma}(\gamma)=C \gamma^{k}
$$

where $C$ is a normalization constant and the function is valid for $\gamma_{1} \leqslant \gamma \leqslant \gamma_{2}$. Later we will discuss other parameterizations of the distribution of Lorentz factors. Here, we assume $\gamma_{1}=5$ and $\gamma_{2}=40$ as this is the range of Lorentz factors observed
Table 4

Parameters of the Beaming Models Described in the Text

\begin{tabular}{lcc}
\hline \hline Parameter & Value & Value \\
\hline$k$ & $-2.03 \pm 0.70$ & $-2.43 \pm 0.11$ \\
$k_{1}$ & $5.1 \pm 0.5^{\mathrm{a}}$ & $5.0 \pm 0.5^{\mathrm{b}}$ \\
$B$ & $3.04 \pm 0.08$ & $3.00 \pm 0.08$ \\
$\gamma_{1}$ & 5 & 5 \\
$\gamma_{2}$ & 40 & 40 \\
$\mathscr{L}_{1}$ & $10^{40}$ & $10^{38}$ \\
$\mathscr{L}_{2}$ & $10^{44}$ & $10^{42}$ \\
$p$ & 4 & 5 \\
$\chi^{2} /$ dof & 1.3 & 1.5 \\
\hline
\end{tabular}

Notes. Parameters without an error estimate were kept fixed during the fitting stage.

a In units of $10^{-23}$.

b In units of $10^{-26}$.

for $\gamma$-loud blazars (e.g., Lähteenmäki \& Valtaoja 2003; Lister et al. 2009b; Savolainen et al. 2010). While the largest intrinsic luminosity $\left(\mathcal{L}_{2}\right)$ can be set free, the lowest one depends on the value of $p$ chosen, i.e., from Equation (21) the beaming factor defines the intrinsic luminosity corresponding to the apparent isotropic luminosity we observe. For $p=4$ and $p=5, \mathcal{L}_{1}$ has to be set equal to $10^{40} \mathrm{erg} \mathrm{s}^{-1}$ and $10^{38} \mathrm{erg} \mathrm{s}^{-1}$, respectively. We set $\mathcal{L}_{2}=10^{4} \mathcal{L}_{1}$, but this choice has hardly any impact on the results.

The free parameters of the problem are the normalization $\left(k_{1}\right)$ and the slope $(B)$ of the intrinsic LF and the slope $k$ of the Lorentz factor distribution. We have fitted Equation (27) to the Fermi LF de-evolved at redshift 0 derived in Section 4.4.1. Figure 12 shows how the best-fit beaming model reproduces the local LF of FSRQs measured by Fermi. From the best fit we derive, for the $p=4$ case, an intrinsic LF slope of $B=3.04 \pm 0.08$ and an index of the Lorentz-factor distribution of $k_{1}=-2.03 \pm 0.70$. The fit values are summarized in Table 4. The Lorentz-factor distribution implies an average Lorentz factor (defined by the range of Lorentz factors given in Table 4) for Fermi blazars of $\gamma=11.7_{-2.2}^{+3.3}$, in reasonable agreement with measured values (see, e.g., Ghisellini et al. 2009). The index of the Lorentz-factor distribution is in agreement with $k_{1} \sim-1.5$ found for the CJ-F survey (Lister \& Marscher 1997). The parameters for the $p=5$ case are very similar to those of the $p=4$ case, but the reduced $\chi^{2}$ is slightly worse (see Table 4 ). Nevertheless, the predictions of the two models are in agreement and we find again that the average bulk Lorentz factor ${ }^{15}$ is $\gamma=10.2_{-2.4}^{+4.8}$. As noted already the extreme Doppler boosting $\left(\delta^{5}\right)$ requires the intrinsic luminosities to be small, i.e., $10^{38} \mathrm{erg} \mathrm{s}^{-1} \leqslant \mathscr{L} \leqslant 10^{42} \mathrm{erg} \mathrm{s}^{-1}$.

From the ratio of the integrals of the beamed and intrinsic LF we derive that the Fermi FSRQs represent only $\approx 0.1 \%$ of the parent population. The average space density of LAT FSRQs (derived from the LF, Section 4.2) is $1.4 \mathrm{Gpc}^{-3}$, implying that the average space density of the parent population is $\sim 1500 \mathrm{Gpc}^{-3}$. Our model also allows us to determine the distribution of jet angles with respect to our line of sight. This is found to peak at $\sim 1$.0 (Figure 13). While FSRQs can still be detected at large (i.e., $\geqslant 10^{\circ}$ ) off-axis angles for reasonably low $\gamma$ factors $(\sim 5-7)$, most FSRQs detected by Fermi are seen at angles less than $5^{\circ}$ from the jet axis. Owing to their larger space density (see Figure 12), misaligned jets produce a non-negligible diffuse emission. From our model it is found that the ratio

\footnotetext{
15 We remind the reader that the distribution of Lorentz factors is limited to be
} in the 5-40 range (see, i.e., Table 4). 


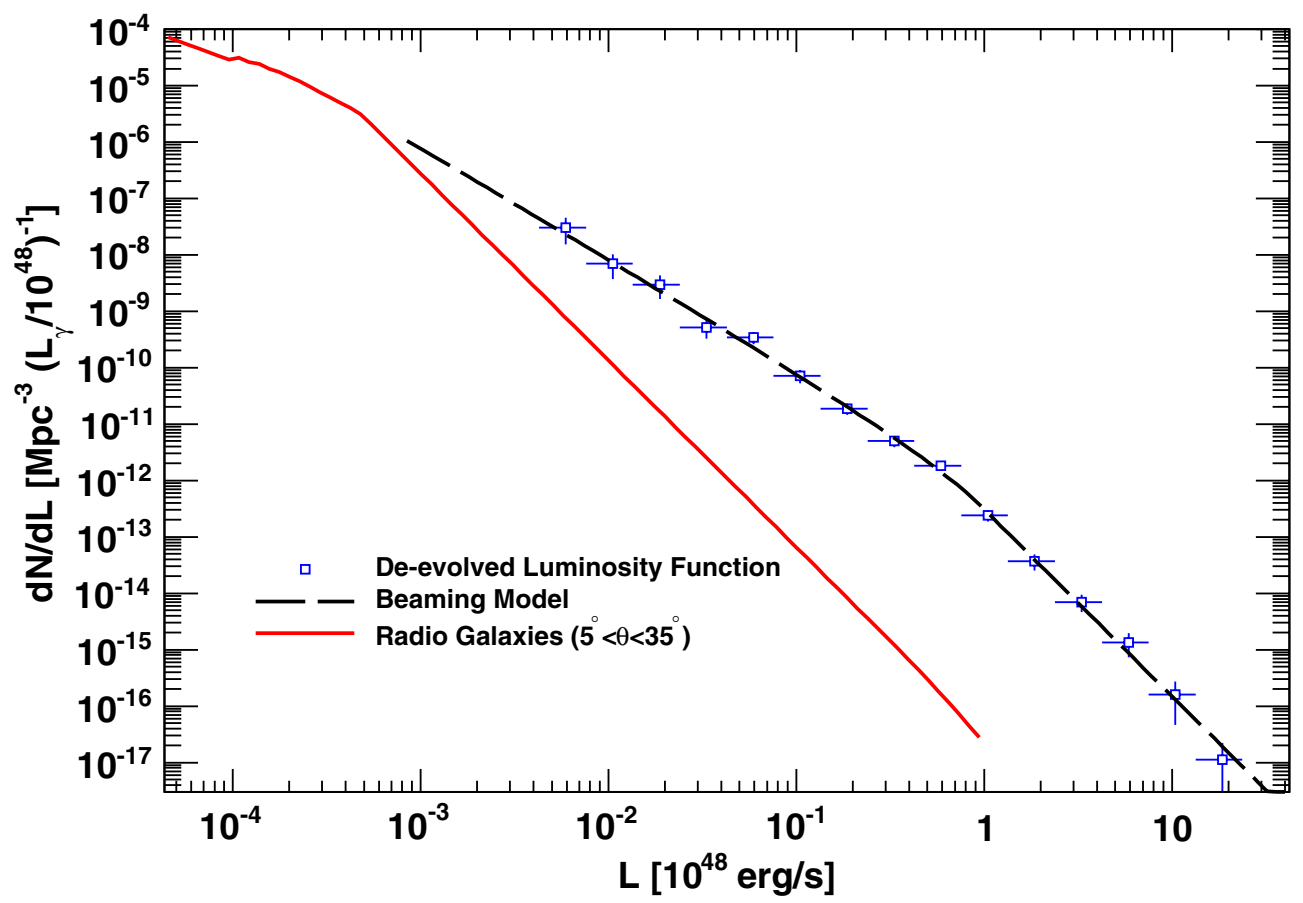

Figure 12. Fermi's LF de-evolved at redshift 0 and the best-fit beaming model (for $p=4$; see the text) described in Section 7. The red continuous line shows the predicted space density of misaligned jets.

(A color version of this figure is available in the online journal.)

between the diffuse emission contribution of misaligned jets and that of blazars (at redshift 0 ) is $\sim 30 \%$. This has obvious consequences for the generation of the IGRB. In fact nearly all of the flux produced by radio galaxies is unresolved, with only two steep-spectrum radio quasars, and two FR II and seven FR I radio galaxies detected with Fermi-LAT (Abdo et al. 2010b). All the results reported above apply to both the $p=4$ and $p=5$ models. Finally we also tested different parameterizations of the distribution of Lorentz factors, i.e., Equation (30). We used a linear, an exponential, and a Gaussian distribution. The only acceptable fit (but statistically worse than the power law) is produced by an exponential distribution of the form: $P(\gamma) \propto e^{-0.07 \gamma}$ which is similar to our power-law model for $\gamma>10$. We thus conclude that the parameterization of the Lorentz factor distribution with a power-law model is a reasonable assumption.

\section{DISCUSSION AND CONCLUSIONS}

In this paper, we examine the properties of $\gamma$-ray-selected FSRQs using data from the Fermi-LAT instrument. Our work relies on a nearly complete, flux-limited sample of 186 FSRQs detected by Fermi at high significance and large Galactic latitude during the first year of operation. This analysis explores several of the properties of FSRQs; here we discuss and summarize our findings.

\subsection{Beamed Luminosity Function}

The redshift-zero LF of Fermi FSRQs is well described by a double power-law model, typical for the LF of both radioquiet and RL AGNs. At mid-to-high luminosities there is good agreement between the Fermi LF and that determined using EGRET data (e.g., Narumoto \& Totani 2006; Inoue et al. 2010). At luminosities $\leqslant 10^{46} \mathrm{erg} \mathrm{s}^{-1}$ the FSRQ LF appears to be slightly flatter than in previous studies.

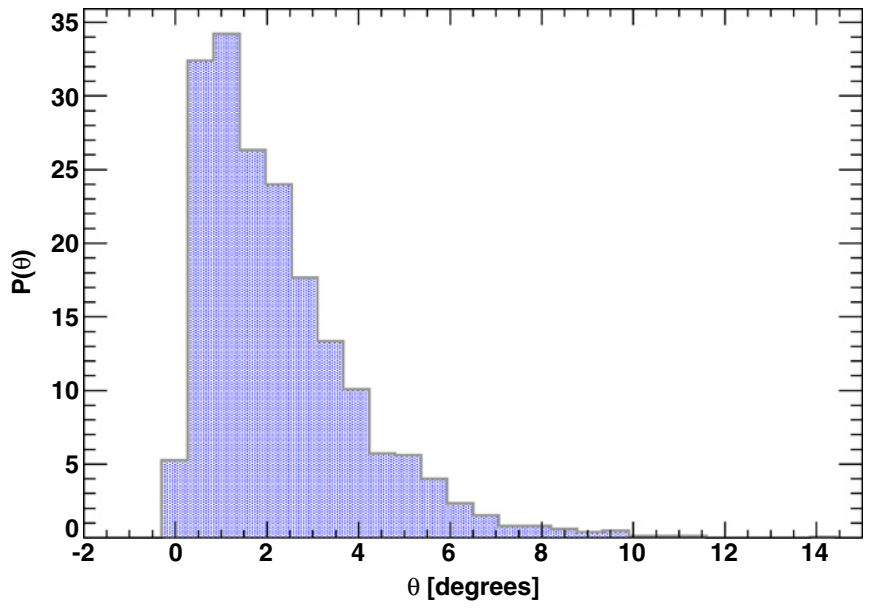

Figure 13. Distribution of viewing angles with respect to the jet axis for Fermi FSRQs.

(A color version of this figure is available in the online journal.)

The space density of LAT-detected FSRQs increases dramatically with redshift, growing by $50-100$ times by $z=1.5$. Describing the evolution of the LF as simple luminosity evolution (PLE model), there are strong indications that the evolution must cut off for $z \geqslant 1.6$. After this redshift, the space density of blazars starts to decrease quickly.

A simple PLE does not fully explain the Fermi data. In particular the source count distribution is not well modeled. Since there is evidence that low- and high-luminosity sources have different redshift peaks, we consider a more sophisticated model where the evolution is primarily in density, but objects with different luminosity are allowed to have different redshift peaks. This so-called LDDE model explains well the evolutionary behavior of (radio-quiet) AGNs selected in the X-ray band (Ueda et al. 2003; Hasinger et al. 2005) and was also suggested by Narumoto \& Totani (2006) to describe the LF of EGRET blazars. 


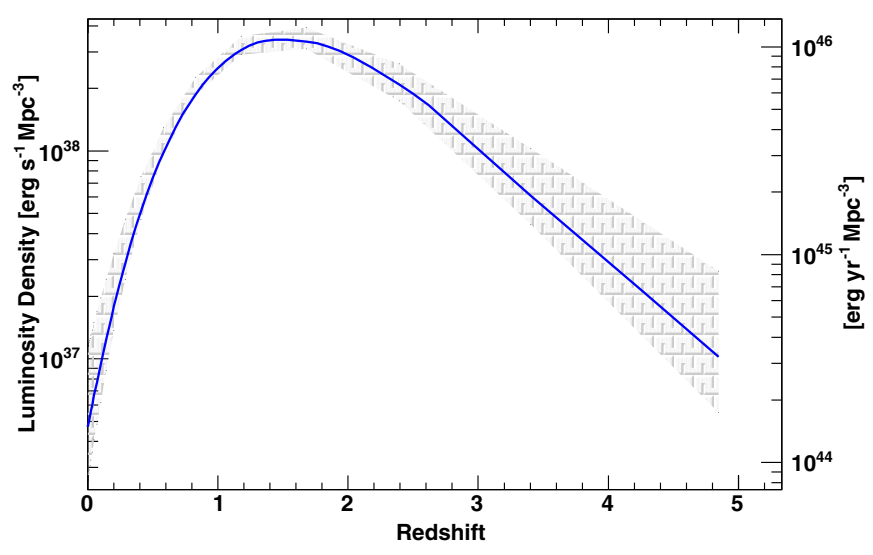

Figure 14. Luminosity density as a function of redshift produced by the Fermi FSRQs. The gray band represents the $1 \sigma$ statistical uncertainty around the bestfit LF model.

(A color version of this figure is available in the online journal.)

The LDDE model provides a good description of the LF of the Fermi FSRQs. We find that the predictions reported in the literature (e.g., Narumoto \& Totani 2006; Inoue et al. 2010; Stecker $\&$ Venters 2011) are not in agreement with the LF of Fermi FSRQs at redshift unity. This is due to the fact that the Fermi FSRQ LF is found to evolve more quickly than the LFs of X-ray-selected AGNs or radio-selected FSRQs. Indeed, the space density of Fermi FSRQs increases by a factor $\sim 150$ between redshifts 0 and 1 while the density increase is at most a factor $\sim 60$ for the models discussed above.

The LDDE model implies that sources with a luminosity of $10^{46} \mathrm{erg} \mathrm{s}^{-1}, 10^{47} \mathrm{erg} \mathrm{s}^{-1}$, and $10^{48} \mathrm{erg} \mathrm{s}^{-1}$ reach their maximum space density at a redshift of $\sim 0.6, \sim 0.9$, and $\sim 1.5$, respectively. It is clear, then, that the most luminous objects, while lower in numbers, appear before the bulk of the (lowluminosity) population. This downsizing in luminosity, where the most luminous objects are found at earlier times, is common to all classes of AGNs (see, e.g., Cowie et al. 1999; Hasinger et al. 2005, and references therein), but is observed here for the first time at gamma-rays. This type of downsizing does not necessarily reflect more conventional downsizing in terms of increased star formation activity in less massive galaxies at late times (Cowie et al. 1996) because the host galaxy and black hole masses are not known, but corresponds to the downsizing behavior observed in submillimeter (sub-mm) galaxies (Wall et al. 2008) even though the underlying astrophysics is different (on the one hand, concerning relativistic jets, and, on the other, dust-enshrouded sub-mm galaxies). The disappearance of quasar-like objects at late times might indicate that accretion efficiency evolves as a function of cosmic time (e.g., Merloni 2004). Sanders et al. (1988) and Di Matteo et al. (2005) propose that the merging of two massive galaxies leads to, in addition to strong star formation activity, a burst of inflow feeding gas to the SMBH and initiates a "quasar-like" phase. Eventually the energy released by the AGN in the form of powerful winds expels the gas, quenches star formation, and starves the AGN. This picture, coupled with the fact that major mergers become increasingly rare at low redshift (e.g., Fakhouri et al. 2010; Kulkarni \& Loeb 2012), may explain why quasars are rare in the local universe.

Figure 14 shows the energy density injected in the universe (e.g., the luminosity density) by FSRQs as function of redshift. This shows a broad peak between a redshift of 1 and 2. A similar behavior is shown by the cosmic star formation history (e.g.,

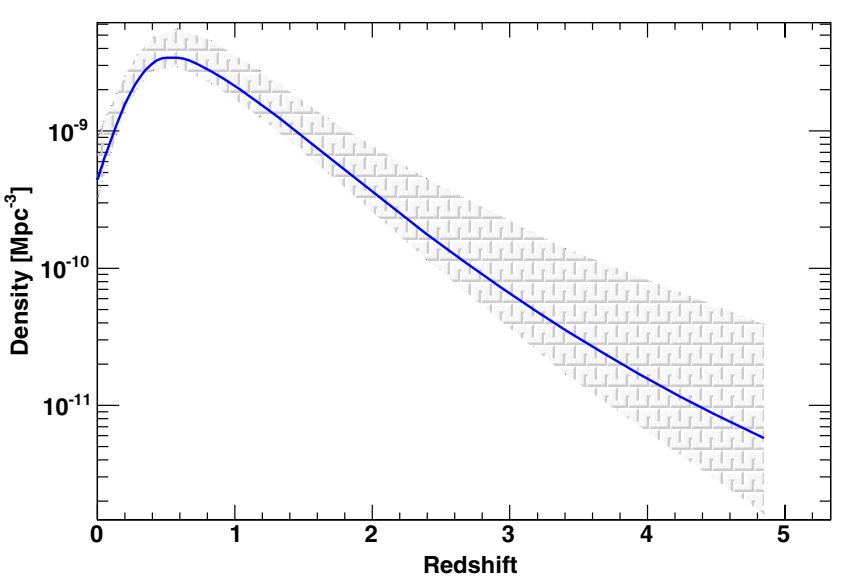

Figure 15. Number density of LAT-detected FSRQs as a function of redshift. The gray band represents the $1 \sigma$ statistical uncertainty around the best-fit LF model.

(A color version of this figure is available in the online journal.)

Hopkins \& Beacom 2006) which peaks around redshifts 1-2. This represents a strong link between the host and the nucleus. A noteworthy fact is the mild evidence for a fast decline in the space density of FSRQs after the redshift peak (see parameter $p_{2}$ in Table 3 and also Figures 4 and 15). The decline seems to be as dramatic as the increase in space density leading up to the redshift peak. For comparison, X-ray-selected samples of AGNs show a much milder decline $\left(p_{2} \approx-1.5\right)$ after the redshift peak (e.g., Ueda et al. 2003; Hasinger et al. 2005; Aird et al. 2010). However, Schmidt et al. (1995) and more recently Silverman et al. (2008) reported evidence for a similarly dramatic decrease in the space density of AGNs.

One factor contributing to this phenomenon is the difficulty for Fermi to detect soft sources (Abdo et al. 2010f). At redshift $\geqslant 3$ the SED peak should move well below the current LAT energy band, making it difficult to probe a population of extremely soft sources. Increasing the effective area at or below $100 \mathrm{MeV}$ may help uncover such a population. Because the rising part of the IC peak is in the hard ( $\geqslant 10 \mathrm{keV}) \mathrm{X}$-ray band, high-redshift objects are more easily selected in this band (see, e.g., the Swift/BAT results in Ajello et al. 2009a). In this case another strategy would be to build a bolometric LF that uses both the $\gamma$-ray and the X-ray-selected samples.

\subsection{The Intrinsic Luminosity Function}

Doppler boosting allows Fermi to detect many blazars when their jet emission is within a few degrees from the line of sight. As shown first by Urry \& Shafer (1984), Doppler boosting is known to alter the shape of the LF. In this paper, we adopted a formalism that allowed us to recover the intrinsic de-beamed LF and to determine the distribution of bulk Lorentz factors for the Fermi FSRQs.

We found that the intrinsic LF can be modeled by a single steep power law with an index of $3.04 \pm 0.08$ in the range of intrinsic luminosities $10^{40} \mathrm{erg} \mathrm{s}^{-1} \leqslant \mathscr{L} \leqslant 10^{44} \mathrm{erg} \mathrm{s}^{-1}$. The break seen in the beamed LF at redshift 0 is thus produced by Doppler boosting. The data cannot be explained by a single, averaged, Lorentz factor, but require a distribution of Lorentz factors. This distribution is found to be compatible with a power law with an index of $-2.03 \pm 0.70$ in the $5 \leqslant \gamma \leqslant 40$ range. This yields the result that the average FSRQ bulk Lorentz factor is $\gamma=11.7_{-2.2}^{+3.3}$, in good agreement with several studies (Ghisellini et al. 2009). Our model is able to predict the distribution of 
viewing angles with respect to the jet axes of Fermi FSRQs. It is found, see Figure 13, that on average FSRQs are seen within an average angle of $\sim 2.3$ from the jet and that most are seen within $5^{\circ}-6^{\circ}$. A few Fermi FSRQ detections may be up to $15^{\circ}$ off-axis (if these have low Doppler factors). Fermidetected FSRQs represent only $\sim 0.1 \%$ of the parent population for randomly pointed jets.

Monitoring observations with the Very Long Baseline Array (VLBA) established that LAT-detected blazars have, on average, significantly faster apparent jet speeds than non-LAT detected blazars (Lister et al. 2009a; Savolainen et al. 2010). Their distribution of Lorentz factors is in good agreement with the results of our analysis. Moreover, they report the distribution of viewing angles with respect to the jet axis for FSRQs detected by LAT. From their study the average viewing angle is $2.9 \pm 0.3$ and all the FSRQs in their sample have $\theta \leqslant 5^{\circ}$. There is thus substantial agreement between the VLBA monitoring observations and the results of our beaming model applied to $\gamma$-ray-only data.

The space density of FR-II radio galaxies (i.e., the putative parent population of FSRQs) is reported to be $\sim 1580 \mathrm{Gpc}^{-3}$ (at $15 \mathrm{GHz}$ ) and $\sim 2200 \mathrm{Gpc}^{-3}$ (at $1.4 \mathrm{GHz}$ ) by Cara \& Lister (2008) and Gendre et al. (2010), respectively. From our study we derive a space density of FSRQ parents of $\sim 1500 \mathrm{Gpc}^{-3}$ in substantial agreement with the numbers above.

Future work may test whether the intrinsic properties of blazars (i.e., Lorentz factor, luminosity, etc.) evolve with redshift. This will likely require larger complete samples and improved modeling of selection effects.

\subsection{Spectral Energy Distribution}

Blazar SEDs are characterized by the typical "two hump" spectrum where the low-energy peak is produced by electrons radiating via synchrotron and the high energy peak is produced via IC scattering off the same synchrotron photons (SSC scenario; Maraschi et al. 1992) and/or external seed photons (EC scenario; Dermer \& Schlickeiser 1993).

In this work, we have combined quasi-simultaneous Swift/BAT and Fermi/LAT data to investigate the empirical properties of the IC component of the SEDs of the FSRQs detected by Fermi. All the SEDs show apparent curvature and have a peak somewhere in the $10 \mathrm{MeV}-1 \mathrm{GeV}$ band. For the FSRQs in our sample, we have determined the peak luminosity of the IC gamma-ray component and the rest-frame peak frequency (or peak energy) at which the IC component reaches its peak luminosity. No correlation is found either between peak luminosities and peak energies or between bolometric luminosities of the IC component and peak energies, as shown in Figure 8 . The existence of such correlation has been claimed in the past for the luminosity and the energy of the synchrotron peak (Ghisellini et al. 1998; Fossati et al. 1998) for a sample of blazars (i.e., FSRQs and BL Lac objects). Thus, it might be that this correlation (if real) exists only when the two families of blazars are joined together and that any correlation for the FSRQs class is washed away by the presence of the additional EC component. Also the lack of correlation of the IC peak luminosity and frequency reveals that FSRQs are, unlike BL Lac objects, part of a population with homogenous properties.

We built average redshift-corrected SEDs in four different luminosity bins. The average SEDs are surprisingly similar as a function of luminosity (and redshift) as Figure 9 testifies. Approximating the SED with a power law with an index 2.4, while not producing the correct shape, allows the reader to compute a $k$-correction useful up to redshift $\approx 2$. Beyond that this approximation is not valid.

\subsection{The Contribution to the Diffuse Background}

This work has important consequences for our understanding of the origins of the diffuse background. As pointed out by several authors (e.g., Inoue \& Totani 2009) and determined in this work, the spectrum of the diffuse emission arising from FSRQs shows curvature, due to the curved SEDs of these objects. We couple our model SED to our LF to predict the FSRQ contribution to the $10 \mathrm{keV}$ to $100 \mathrm{GeV}$ diffuse background. FSRQs produce $\sim 10 \%$ of the cosmic diffuse emission in the $1 \mathrm{MeV}-10 \mathrm{GeV}$ band.

Because of its good sensitivity Fermi has already resolved as much as $50 \%$ of the total flux from FSRQs in the $100 \mathrm{MeV}-100 \mathrm{GeV}$ band. Our analysis indicates that the contribution of unresolved FSRQs to the IGRB (Abdo et al. 2010e) is $9.66_{-1.09}^{+1.67} \times 10^{-7}$ photons $\mathrm{cm}^{-2} \mathrm{~s}^{-1} \mathrm{sr}^{-1}$ and thus only $9.3_{-1.0}^{+1.6} \%$ $( \pm 3 \%$ systematic uncertainty) of the intensity of the IGRB. This analysis is in good agreement with the results reported by Abdo et al. (2010f) except above $10 \mathrm{GeV}$ where the use of a simple power law for the spectra of FSRQs was inadequate.

Our results appear in reasonably good agreement with those of Inoue et al. (2010) and of Inoue \& Totani (2011), particularly in terms of spectral shape of the diffuse emission arising from FSRQs. These authors rely on the EGRET sample, which contains both FSRQs and BL Lac objects, so it is not surprising that their estimates of the blazar contribution to the EGRB are larger, by a factor $\sim 2$ at $1 \mathrm{GeV}$, than ours. Finally, our estimate reported above is in good agreement with the results of Dermer (2007) that predicted that FSRQs would produce $\approx 10 \%-15 \%$ of the $\gamma$-ray background.

LAT-detected FSRQs represent only $\sim 0.1 \%$ of the parent population (see Section 7) and thus it is reasonable to expect that misaligned jets, although less luminous but more numerous, give a non-negligible contribution to the diffuse background. Our beaming model allowed us to explore this scenario. It is found that misaligned relativistic jets contribute $\sim 30 \%$ of the diffuse flux from the FSRQs class at redshift 0. If the Lorentz factor distribution does not change with redshift then the contribution of unresolved FSRQs and their misaligned siblings might be around $\sim 2.0 \times 10^{-6}$ photons $\mathrm{cm}^{-2} \mathrm{~s}^{-1}$ and thus $\sim 20 \%$ of the IGRB. Recently, Inoue \& Totani (2011) predicted that radio galaxies of both the FR-I and FR-II types might be able to account for $\sim 25 \%$ of the intensity of the IGRB. In our work we found that FR-II alone could in principle (see above caveat) produce $\sim 10 \%$ of the IGRB. It can be envisaged that once also the contribution to the IGRB of BL Lac objects and their parents will be established, the total $\gamma$-ray emission from relativistic jets might account for some $\sim 40 \%-50 \%$ of the intensity of the IGRB. Moreover, star-forming galaxies at lower energies are likely to remedy some of the differences between the intensity of the IGRB and the contribution from FSRQs shown in Figure 11, though contributions from other source classes may be required to explain the entire IGRB spectrum.

The authors acknowledge a very constructive report from an expert referee. M.A. acknowledges Y. Inoue and T. Venters for providing their data in electronic form and for interesting discussions about the origin of the IGRB. M.A. acknowledges support from NASA grant NNH09ZDA001N for the study of the origin of the Isotropic Gamma-ray Background. R.W.R. acknowledges 
NASA grant NNX08AW30G and extensive consultation with the OVRO Fermi group.

The Fermi-LAT Collaboration acknowledges generous ongoing support from a number of agencies and institutes that have supported both the development and the operation of the LAT as well as scientific data analysis. These include the National Aeronautics and Space Administration and the Department of Energy in the United States, the Commissariat à l'Energie Atomique and the Centre National de la Recherche Scientifique/Institut National de Physique Nucléaire et de Physique des Particules in France, the Agenzia Spaziale Italiana and the Istituto Nazionale di Fisica Nucleare in Italy, the Ministry of Education, Culture, Sports, Science and Technology (MEXT), High Energy Accelerator Research Organization (KEK), and Japan Aerospace Exploration Agency (JAXA) in Japan, and the K. A. Wallenberg Foundation, the Swedish Research Council, and the Swedish National Space Board in Sweden. Additional support for science analysis during the operations phase is gratefully acknowledged from the Istituto Nazionale di Astrofisica in Italy and the Centre National d'Études Spatiales in France.

Facilities: Fermi (LAT), Swift (BAT)

\section{APPENDIX}

\section{SYSTEMATIC UNCERTAINTIES}

The sources of systematic uncertainties in this analysis are incompleteness (i.e., missing redshifts), detection efficiency, blazar variability, and EBL. A detailed discussion of some of these problems was already given in Abdo et al. (2010f). Incompleteness in our sample is very small and introduces no appreciable systematic uncertainty as shown in Section 4.3.

\section{A.1. Detection Efficiency}

The detection efficiency used to determine the sky area surveyed by Fermi at any given flux is very important in this analysis (see Abdo et al. 2010f, for a detailed discussion). The detection efficiency used in this work was derived in Abdo et al. (2010f) under the assumption that the blazar spectra can be approximated by a power law. While this might be true over a small energy band, it becomes a problematic assumption over the full $100 \mathrm{MeV}-100 \mathrm{GeV}$ band covered by LAT. In this Appendix, we estimate directly the systematic uncertainties connected to this hypothesis. We performed three end-to-end Monte Carlo simulations of the Fermi sky (see Abdo et al. 2010 for details), assigning randomly a curved spectrum to each source. These spectra are extracted from a library created using the $\sim 100$ observed spectra derived in Section 5 varying the parameters of the measured spectra within their errors. The simulations were then analyzed to derive the detection efficiency reported in Figure 16. In particular in order to detect a source, an ML fit with a power-law spectrum is performed. This is done in order to reproduce the inherent systematic uncertainty of fitting the curved spectrum of a source with a power law (Abdo et al. 2010a).

Figure 16 shows the detection efficiency for a sample like that used in this analysis. Two aspects are noteworthy. First the efficiency at $F_{100}=10^{-8}$ photons $\mathrm{cm}^{-2} \mathrm{~s}^{-1}$ (i.e., the lowest flux of this analysis by construction) is $\sim 0.02$ with a typical uncertainty of $\pm 5 \times 10^{-3}$ dictated by the small number of sources detected in our simulations at the lowest fluxes. Second, at fluxes around $F_{100} \approx 10^{-7}$ photons $\mathrm{cm}^{-2} \mathrm{~s}^{-1}$ the detection efficiency becomes larger than 1.0. This effect is due to the fact

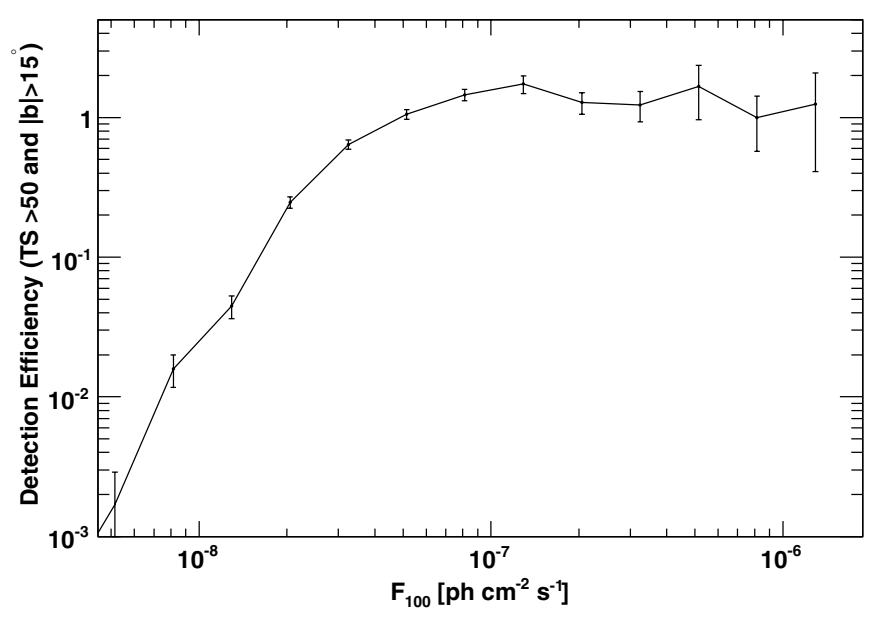

Figure 16. Detection efficiency as a function of flux for a population of sources with curved spectra similar to those of FSRQs determined in Section 5.

that fitting a curved spectrum source with a power law yields to an overestimate of the source flux by a factor $\sim 10 \%$ (see also Figure 8 in Abdo et al. 2010f). Since Figure 16 is built as the ratio (in a given bin) of the number of sources detected with a given flux to the number of simulated sources with that flux, the effect mentioned above leads to a detection efficiency $>1.0$.

In order to test the level of systematic uncertainty we derived the LF using the detection efficiency reported in Figure 16 (instead of using the detection efficiency curves reported in Abdo et al. 2010f and used throughout Section 4). Given the "small" number of sources detected in the three simulations, it was not possible to derive a two-dimensional detection efficiency as a function of flux and spectral index (like that used in Section 4 and derived for power-law sources in Abdo et al. 2010f). For this reason the parameters of the distribution of photon indices of the FSRQ class cannot be derived from the analysis of the LF. As apparent from Table 3 most parameters of the LF derived in this section and those derived in Section 4.2 are compatible within their statistical errors. The only parameter for which the difference is slightly larger than the statistical errors is $\alpha$. The parameter $\alpha$ governs the trend of the redshift peak with luminosity and while its statistical error is in both case 0.03 , the systematic error appears to be 0.05 . This has very little impact on the analysis and the results of the previous sections are fully confirmed and robust against variations of the detection efficiency curve. As a further proof, the points of the de-evolved LF in Figures 5 and 6 were computed using the detection efficiency of Figure 16 while the shaded error region was computed using the model LF derived in Section 4.2 that uses the detection efficiency for power-law sources.

We performed an additional test by shifting the detection efficiency curve in Figure 16 to fluxes $10 \%$ brighter than measured. The rightward shift is most dramatic as it increases the magnitude of the correction at faint fluxes. The shift is performed in order to account for uncertainties in the determination of the detection efficiency. The parameters of the LF are all consistent within statistical uncertainty with those found in this and the previous sections and reported in Table 3. The index of the lowluminosity slope of the LF becomes slightly steeper (i.e., $\gamma_{1}=$ $0.47 \pm 0.18)$, and this yields a slightly larger contribution to the IGRB from FSRQs. We thus consider the typical systematic uncertainty connected to the estimate of the contribution to the IGRB to be $\sim 3 \%$ of the IGRB $100 \mathrm{MeV}-100 \mathrm{GeV}$ intensity. 


\section{A.2. Variability}

It is well known that blazars are inherently variable objects with variability in flux of up to a factor 10 or more. Throughout this work only average quantities (i.e., mean flux, mean luminosity, and mean photon index) are used.

It is not straightforward to determine how blazar variability affects the analysis presented here. While the variability patterns and amplitudes of blazars as a class are still not known both Abdo et al. (2010c) and Ackermann et al. (2011) presented a detailed analysis of the variability of the brightest Fermi blazars. They report that the variability amplitude of the FSRQ class is generally larger than that of the BL Lac population. However, most sources (either bright or faint ones) exceed their average flux for less than $5 \%-20 \%$ of the monitored time (i.e., respectively, 11 months or 2 years). This drastically reduces the possibility that FSRQs (or blazars more in general) are detected because of a few bright flaring episodes. The effect of highamplitude variability connected with a rising density of sources at smaller flux might contaminate samples, as the one used here, with objects that formally should not be included. However, because of what has been reported above and the flatness of the FSRQs source count distribution (see Abdo et al. 2010f; Ackermann et al. 2011, and Figure 2) this effect is very likely marginal.

Another, smaller, problem is connected to the dependence of the effective area on the direction of the incoming photon and the LAT detector frame. ${ }^{16}$ Short intense flares detected during favorable conditions (i.e., on axis and at azimuthal angles of $\sim 0^{\circ}$, $\sim 90^{\circ}, \sim 180^{\circ}$, or $\sim 270^{\circ}$ ) might lead to a higher TS, increasing the likelihood of source detection. However, because of Fermi's continuous scanning of the sky and because most flares are observed to last 10 days or longer (Abdo et al. 2010c), the effect above has negligible influence on the analysis presented here.

Finally, we believe that variability does not hamper the results of this analysis. Using even longer integration times (e.g., two or three years) will be the most efficient way to confirm the results of this analysis and dilute the effect of blazar variability.

\section{A.3. Extragalactic Background Light}

Uncertainty in the level of the EBL, in particular at medium to high redshift, might in principle affect our analysis. Energetic $\gamma$-rays from FSRQs at high redshifts might be absorbed by the EBL and if this effect is not taken into account the source-frame luminosity would be underestimated. This would lead to wrong estimates of the space densities of FSRQs. However, we believe that this uncertainty is negligible.

The uncertainty in the level of the EBL would impact the estimate of the $k$-correction, which allows us to determine the source-frame luminosities. As shown in Figure 10, neglecting the EBL at once and adopting a simple power-law spectrum with a photon index of 2.4 (instead of the average SED determined in Section 5) introduces an uncertainty of $\leqslant 4 \%$ on the value of the $k$-correction at $z=3$. Since all the Fermi FSRQs are detected within this redshift, this uncertainty produces no appreciable impact on the determination of the LF.

\section{REFERENCES}

Abdo, A. A., Ackermann, M., Ajello, M., et al. 2009, ApJ, 700, 597 Abdo, A. A., Ackermann, M., Ajello, M., et al. 2010a, ApJS, 188, 405

\footnotetext{
16 See e.g., http://www.slac.stanford.edu/exp/glast/groups/canda/lat
} Performance.htm and Atwood et al. (2009).
Abdo, A. A., Ackermann, M., Ajello, M., et al. 2010b, ApJ, 720, 912 Abdo, A. A., Ackermann, M., Ajello, M., et al. 2010c, ApJ, 722, 520 Abdo, A. A., Ackermann, M., Ajello, M., et al. 2010d, ApJ, 710, 1271 Abdo, A. A., Ackermann, M., Ajello, M., et al. 2010e, Phys. Rev. Lett., 104, 101101

Abdo, A. A., Ackermann, M., Ajello, M., et al. 2010f, ApJ, 720, 435 Abdo, A. A., Ackermann, M., Ajello, M., et al. 2010g, ApJ, 715, 429 Ackermann, M., Ajello, M., Allafort, A., et al. 2011, ApJ, 743, 171

Aird, J., Nandra, K., Laird, E. S., et al. 2010, MNRAS, 401, 2531

Ajello, M., Costamante, L., Sambruna, R. M., et al. 2009a, ApJ, 699, 603

Ajello, M., Greiner, J., Kanbach, G., et al. 2008a, ApJ, 678, 102

Ajello, M., Greiner, J., Sato, G., et al. 2008b, ApJ, 689, 666

Ajello, M., Rau, A., Greiner, J., et al. 2008c, ApJ, 673, 96

Ajello, M., Rebusco, P., Cappelluti, N., et al. 2009b, ApJ, 690, 367

Ajello, M., Rebusco, P., Cappelluti, N., et al. 2010, ApJ, 725, 1688

Atwood, W. B., Abdo, A. A., Ackermann, M., et al. 2009, ApJ, 697, 1071

Avni, Y. 1976, ApJ, 210, 642

Barthelmy, S. D., Barbier, L. M., Cummings, J. R., et al. 2005, Space Sci. Rev., 120,143

Beckmann, V., Engels, D., Bade, N., \& Wucknitz, O. 2003, A\&A, 401, 927

Begelman, M. C., Volonteri, M., \& Rees, M. J. 2006, MNRAS, 370, 289

Bhattacharya, D., Sreekumar, P., \& Mukherjee, R. 2009, Res. Astron. Astrophys., 9, 85

Blandford, R. D., \& Znajek, R. L. 1977, MNRAS, 179, 433

Caccianiga, A., Maccacaro, T., Wolter, A., Della Ceca, R., \& Gioia, I. M. 2002, ApJ, 566, 181

Cara, M., \& Lister, M. L. 2008, ApJ, 674, 111

Chiang, J., Fichtel, C. E., von Montigny, C., Nolan, P. L., \& Petrosian, V. 1995, ApJ, 452, 156

Chiang, J., \& Mukherjee, R. 1998, ApJ, 496, 752

Cowie, L. L., Songaila, A., \& Barger, A. J. 1999, AJ, 118, 603

Cowie, L. L., Songaila, A., Hu, E. M., \& Cohen, J. G. 1996, AJ, 112, 839

Croton, D. J., Springel, V., White, S. D. M., et al. 2006, MNRAS, 365, 11

Cusumano, G., La Parola, V., Segreto, A., et al. 2010, A\&A, 524, A64

Della Ceca, R., Caccianiga, A., Severgnini, P., et al. 2008, A\&A, 487, 119

Dermer, C. D. 1995, ApJ, 446, L63

Dermer, C. D. 2007, ApJ, 659, 958

Dermer, C. D., \& Schlickeiser, R. 1993, ApJ, 416, 458

Di Matteo, T., Springel, V., \& Hernquist, L. 2005, Nature, 433, 604

Dotti, M., Colpi, M., Haardt, F., \& Mayer, L. 2007, MNRAS, 379, 956

Dunlop, J. S., \& Peacock, J. A. 1990, MNRAS, 247, 19

Escala, A., Larson, R. B., Coppi, P. S., \& Mardones, D. 2004, ApJ, 607, 765

Fakhouri, O., Ma, C.-P., \& Boylan-Kolchin, M. 2010, MNRAS, 406, 2267

Fan, X., Strauss, M. A., Schneider, D. P., et al. 2003, AJ, 125, 1649

Fichtel, C. E., Hartman, R. C., Kniffen, D. A., et al. 1975, ApJ, 198, 163

Fossati, G., Maraschi, L., Celotti, A., Comastri, A., \& Ghisellini, G. 1998, MNRAS, 299, 433

Franceschini, A., Rodighiero, G., \& Vaccari, M. 2008, A\&A, 487, 837

Gehrels, N., Chincarini, G., Giommi, P., et al. 2004, ApJ, 611, 1005

Gendre, M. A., Best, P. N., \& Wall, J. V. 2010, MNRAS, 404, 1719

Ghisellini, G., Celotti, A., Fossati, G., Maraschi, L., \& Comastri, A. 1998, MNRAS, 301, 451

Ghisellini, G., Tavecchio, F., \& Ghirlanda, G. 2009, MNRAS, 399, 2041

Giommi, P., \& Padovani, P. 1994, MNRAS, 268, L51

Hartman, R. C., Bertsch, D. L., Bloom, S. D., et al. 1999, ApJS, 123, 79

Hasinger, G., Miyaji, T., \& Schmidt, M. 2005, A\&A, 441, 417

Healey, S. E., Romani, R. W., Cotter, G., et al. 2008, ApJS, 175, 97

Healey, S. E., Romani, R. W., Taylor, G. B., et al. 2007, ApJS, 171, 61

Hogg, D. W. 1999, arXiv:astro-ph/9905116

Hopkins, A. M., \& Beacom, J. F. 2006, ApJ, 651, 142

Inoue, Y. 2011, ApJ, 733, 66

Inoue, Y., \& Totani, T. 2009, ApJ, 702, 523

Inoue, Y., \& Totani, T. 2011, ApJ, 728, 73

Inoue, Y., Totani, T., \& Mori, M. 2010, PASJ, 62, 1005

Kauffmann, G., \& Haehnelt, M. 2000, MNRAS, 311, 576

Keshet, U., Waxman, E., \& Loeb, A. 2004, J. Cosmol. Astropart. Phys., JCAP04(2004)006

Kraushaar, W. L., Clark, G. W., Garmire, G. P., et al. 1972, ApJ, 177, 341

Kulkarni, G., \& Loeb, A. 2012, MNRAS, 422, 1306

La Franca, F., \& Cristiani, S. 1997, AJ, 113, 1517

La Franca, F., Fiore, F., Comastri, A., et al. 2005, ApJ, 635, 864

Lähteenmäki, A., \& Valtaoja, E. 2003, ApJ, 590, 95

Lister, M. L. 2003, ApJ, 599, 105

Lister, M. L., Cohen, M. H., Homan, D. C., et al. 2009a, AJ, 138, 1874

Lister, M. L., Homan, D. C., Kadler, M., et al. 2009b, ApJ, 696, L22

Lister, M. L., \& Marscher, A. P. 1997, ApJ, 476, 572

Maraschi, L., Ghisellini, G., \& Celotti, A. 1992, ApJ, 397, L5 
Marshall, H. L., Tananbaum, H., Avni, Y., \& Zamorani, G. 1983, ApJ, 269, 35 Massaro, E., Giommi, P., Leto, C., et al. 2009, A\&A, 495, 691

Mattox, J. R., Bertsch, D. L., Chiang, J., et al. 1996, ApJ, 461, 396

Mayer, L., Kazantzidis, S., Escala, A., \& Callegari, S. 2010, Nature, 466, 1082

Merloni, A. 2004, MNRAS, 353, 1035

Miyaji, T., Hasinger, G., \& Schmidt, M. 2001, A\&A, 369, 49

Mücke, A., \& Pohl, M. 2000, MNRAS, 312, 177

Narumoto, T., \& Totani, T. 2006, ApJ, 643, 81

Nieppola, E., Valtaoja, E., Tornikoski, M., Hovatta, T., \& Kotiranta, M. 2008, A\&A, 488, 867

Nolan, P., Abdo, A. A., Ackermann, M., et al. 2012, ApJS, 199, 31

Padovani, P., Giommi, P., Landt, H., \& Perlman, E. S. 2007, ApJ, 662, 182

Rector, T. A., Stocke, J. T., Perlman, E. S., Morris, S. L., \& Gioia, I. M. 2000, AJ, 120,1626

Romani, R. W. 2006, AJ, 132, 1959

Sanders, D. B., Soifer, B. T., Elias, J. H., et al. 1988, ApJ, 325, 74

Savolainen, T., Homan, D. C., Hovatta, T., et al. 2010, A\&A, 512, A24

Schmidt, M. 1968, ApJ, 151, 393
Schmidt, M., Schneider, D. P., \& Gunn, J. E. 1995, AJ, 110, 68 Shaw, M. S., Romani, R. W., Cotter, G., et al. 2012, ApJ, 748, 49 Silverman, J. D., Green, P. J., Barkhouse, W. A., et al. 2008, ApJ, 679, 118

Sreekumar, P., Bertsch, D. L., Dingus, B. L., et al. 1998, ApJ, 494, 523

Stecker, F. W., Malkan, M. A., \& Scully, S. T. 2006, ApJ, 648, 774

Stecker, F. W., \& Salamon, M. H. 1996, ApJ, 464, 600

Stecker, F. W., \& Venters, T. M. 2011, ApJ, 736, 40

Tueller, J., Mushotzky, R. F., Barthelmy, S., et al. 2008, ApJ, 681, 113

Ueda, Y., Akiyama, M., Ohta, K., \& Miyaji, T. 2003, ApJ, 598, 886

Urry, C. M., \& Padovani, P. 1991, ApJ, 371, 60

Urry, C. M., \& Shafer, R. A. 1984, ApJ, 280, 569

Volonteri, M., \& Rees, M. J. 2005, ApJ, 633, 624

Wall, J. 2008, arXiv:0807.3792

Wall, J. V., Jackson, C. A., Shaver, P. A., Hook, I. M., \& Kellermann, K. I. 2005, A\&A, 434, 133

Wall, J. V., Pope, A., \& Scott, D. 2008, MNRAS, 383, 435

Willott, C. J., Delorme, P., Reylé, C., et al. 2010, AJ, 139, 906

Wolter, A., \& Celotti, A. 2001, A\&A, 371, 527

Wyithe, J. S. B., \& Loeb, A. 2003, ApJ, 595, 614 